\title{
A little theory of everything, with heavy neutral leptons
}

\author{
James Cline, ${ }^{a}$ Matteo Puel $^{a}$ and Takashi Toma ${ }^{b}$ \\ ${ }^{a}$ McGill University, Department of Physics, \\ 3600 University St., Montréal, QC H3A2T8 Canada \\ ${ }^{b}$ Institute of Liberal Arts and Science, Kanazawa University, Kakuma-machi, \\ Kanazawa, Ishikawa 920-1192 Japan \\ E-mail: jcline@physics.mcgill.ca, matteo.puel@mail.mcgill.ca, \\ toma@staff .kanazawa-u.ac.jp
}

ABSTRACT: Recently a new model of "Affleck-Dine inflation" was presented, that produces the baryon asymmetry from a complex inflaton carrying baryon number, while being consistent with constraints from the cosmic microwave background. We adapt this model such that the inflaton carries lepton number, and communicates the lepton asymmetry to the standard model baryons via quasi-Dirac heavy neutral leptons (HNLs) and sphalerons. One of these HNLs, with mass $\lesssim 4.5 \mathrm{GeV}$, can be (partially) asymmetric dark matter (DM), whose asymmetry is determined by that of the baryons. Its stability is directly related to the vanishing of the lightest neutrino mass. Neutrino masses are generated by integrating out heavy sterile neutrinos whose mass is above the inflation scale. The model provides an economical origin for all of the major ingredients missing from the standard model: inflation, baryogenesis, neutrino masses, and dark matter. The HNLs can be probed in fixed-target experiments like $\mathrm{SHiP}$, possibly manifesting $N-\bar{N}$ oscillations. A light singlet scalar, needed for depleting the DM symmetric component, can be discovered in beam dump experiments and searches for rare decays, possibly explaining anomalous events recently observed by the KOTO collaboration. The DM HNL is strongly constrained by direct searches, and could have a cosmologically interesting self-interaction cross section.

Keywords: Cosmology of Theories beyond the SM, Neutrino Physics

ARXIV EPRINT: 2001.11505 


\section{Contents}

1 Introduction 1

2 Model 3

3 Nonstandard leptogenesis and DM relic density 4

3.1 Sharing and preserving the asymmetry 5

$\begin{array}{lll}3.2 & \text { DM asymmetric abundance and maximum mass } & 6\end{array}$

$\begin{array}{lll}3.3 & \text { Dark matter annihilation and relic density } & 7\end{array}$

3.3.1 $N^{\prime} \bar{N}^{\prime} \rightarrow s s$ annihilation $\quad 8$

3.3.2 $\quad N^{\prime} \bar{N}^{\prime} \rightarrow$ SM annihilation $\quad 9$

4 Neutrino properties and HNL constraints 10

$\begin{array}{lll}4.1 & \text { Explicit } \eta_{\nu} \text { and HNL mixings } & 10\end{array}$

$\begin{array}{lll}\text { 4.1.1 Unitarity constraints for } m_{s}<M_{N} \text { case } & 11\end{array}$

4.1.2 Laboratory constraints for $m_{s}>M_{N}$ case 13

$\begin{array}{lll}4.2 & N-\bar{N} \text { oscillations } & 15\end{array}$

$\begin{array}{lll}4.3 & \text { Weak HNL decays } & 15\end{array}$

$\begin{array}{lll}4.4 & \text { Entropy and energy injection by late } N \text { decays } & 16\end{array}$

$\begin{array}{ll}4.5 & \text { Lepton flavor violation bounds } \\ & 17\end{array}$

5 Constraints on the singlet $\quad 18$

$\begin{array}{lll}6 & \text { DM direct/indirect detection and self-interactions } & 19\end{array}$

$\begin{array}{lll}6.1 & \text { DM-nucleon scattering } & 19\end{array}$

6.2 DM indirect detection $\quad 21$

6.3 DM self-interactions 23

$\begin{array}{lll}7 & \text { Naturalness } & 24\end{array}$

8 Conclusions 25

$\begin{array}{ll}\text { A Decay rate for } N_{i} \rightarrow \nu \ell^{+} \ell^{-} & 27\end{array}$

\section{Introduction}

The standard model (SM) of particle physics is noted for being incomplete in numerous ways. It could be argued that the most urgently missing elements are an inflaton (or other source of primordial density perturbations), a mechanism for baryogenesis, dark matter (DM), and the origin of neutrino masses, since all of these relate to directly observed 
phenomena as opposed to problems of naturalness. It is tempting to seek relatively simple new physics models that can simultaneously address several of the missing pieces, or perhaps all. ${ }^{1}$

A notable example is the $\nu \mathrm{MSM}[2,3]$, in which light sterile neutrinos can accomplish leptogenesis and provide a dark matter candidate while giving neutrino masses. Higgs inflation [4] can be invoked in this framework without needing any additional particles. A similar mechanism of getting an inflationary phase was also implemented in the scotogenic model $[5,6]$ to simultaneously explain inflation, dark matter, baryogenesis and neutrino masses, by introducing a scalar inert doublet coupled non-minimally to gravity and three right-handed neutrinos. Another example is the SMASH model [7] that assumes heavy right-handed neutrinos to explain neutrino mass and thermal leptogenesis, while introducing minimal extra matter content to produce axions as dark matter and a solution to the strong CP problem. The extra scalar field needed for breaking Peccei-Quinn symmetry can combine with the Higgs to give two-field inflation in the early universe. The idea of explaining neutrino masses, baryon asymmetry, dark matter, inflation and solving the strong CP problem using three right-handed neutrinos and the extra fields of the KSVZ axion model [8] was originally presented in ref. [9]. There, the Higgs field was identified as the inflaton and the electroweak vacuum was shown to be stable for several choices of the model parameters. The problem of Higgs inflation, which is known to reduce the scale of perturbative unitarity breaking well below the Planck scale, was addressed by coupling the Higgs field nonminimally to gravity [10].

In the present work we suggest another way of completing the standard model, that does not rely upon leptogenesis as usually defined (through the CP-violating out-ofequilibrium decays of heavy neutrinos). The starting point is a model of inflation in which the Affleck-Dine mechanism [11] for creating a particle asymmetry occurs during inflation [12]. The asymmetry is originally stored in a complex inflaton field, that has the Lagrangian

$$
\mathcal{L}=\frac{m_{P}^{2}}{2} R\left(1+2 \xi|\phi|^{2}\right)+|\partial \phi|^{2}-m_{\phi}^{2}|\phi|^{2}-\lambda|\phi|^{4}-i \lambda^{\prime}\left(\phi^{4}-\phi^{* 4}\right)
$$

(where $m_{P}$ is the reduced Planck scale) including a nonminimal coupling to gravity, needed to flatten the potential at large $|\phi|$, which makes the inflationary predictions compatible with Planck constraints [13]. In ref. [12] we assumed that $\phi$ carried baryon number, which was transferred to the SM quarks through colored scalar mediators. Here we consider the case where $\phi$ carries lepton number, hence giving a new mechanism of leptogenesis. As usual, the resulting lepton asymmetry is transmitted to the baryons through the sphaleron interactions of the SM.

The challenge for such an approach is to find a way of transferring the lepton asymmetry from $\phi$ to the SM without it being washed out by the lepton-violating effects associated with neutrino mass generation. Indeed, if $\phi$ decays to heavy right-handed neutrinos that have large Majorana masses, the asymmetry gets washed out immediately and the situation

\footnotetext{
${ }^{1}$ Ref. [1] provides a recent attempt in this direction, in which an inflaton-like field is present, although the details of inflation are not yet worked out.
} 
reverts to standard leptogenesis being required. This suggests that $\phi$ should decay into quasi-Dirac neutrino mediators $N_{i}$, that mix with the SM neutrinos to transmit the asymmetry. Among the $N_{i}$ mediators, one can be stable and constitute a species of asymmetric dark matter, getting its relic density (partly) from the initial lepton asymmetry. The $N_{i}$ are an example of heavy neutral leptons (HNLs), a class of hypothetical particles that is being widely studied both theoretically and by upcoming experiments such as SHiP [14], MATHUSLA [15], FASER [16] and CODEX-b [17].

To deplete the symmetric component of the DM to a viable level, it is necessary to introduce a light mediator, which we take to be a scalar singlet $s$, so that $N_{i} \bar{N}_{i} \rightarrow$ ss annihilations are sufficiently strong. The DM can be fully or partially asymmetric depending on the coupling strength $g_{s}$. We will show that this interaction has interesting implications for direct detection, and for hints of anomalous rare $K_{L} \rightarrow \pi^{0}+$ invisible decays that have recently been reported by the KOTO experimental collaboration [18].

In our proposal, the HNLs do not explain the origin of light neutrino masses, but we hypothesize that their couplings to the SM $\nu$ 's are related to those of the superheavy Majorana $\nu_{R}$ 's that generate seesaw masses, by a principle similar to minimal flavor violation (MFV) [19]. The setup thereby also addresses the origin of neutrino mass and relates the HNL couplings to it in an essential way. Moreover a direct link is made between the stability of the dark matter candidate and the masslessness of the lightest SM neutrino.

In section 2 we specify the structure of couplings of the HNLs to the inflaton and $\mathrm{SM}$ particles, and its relation to neutrino mass generation. In section 3 we discuss constraints on the couplings such that the lepton asymmetry from inflation is transferred to the SM particles without being washed out. It is shown how the resulting baryon asymmetry determines the dark matter asymmetry and its mass. The relations between light $\nu$ properties and the HNL couplings are presented in section 4, and consequent predictions for the phenomenology of the HNLs. In section 5 we compile the experimental limits on the light singlet $s$, and identify a region of parameter space where the KOTO anomaly can be reconciled with DM direct detection limits. The latter are considered in detail in section 6, where we also treat the DM self-interactions and discuss possible DM indirect detection constraints. The technical naturalness of our setup is demonstrated in section 7 , followed by conclusions in section 8. In appendix A we derive the exact width for HNL decay into different-flavor charged leptons, which was given only in approximate form in previous papers.

\section{Model}

We assume the inflaton carries lepton number 2 (more correctly, $B-L=-2$ since $B-L$ symmetry is not broken by electroweak sphalerons), and couples to $N_{N}$ flavors of quasiDirac HNLs as

$$
g_{\phi} \phi \bar{N}_{L, i} N_{L, i}^{c}+g_{\phi} \phi \bar{N}_{R, i} N_{R, i}^{c}+\text { H.c. }
$$

$N_{N}$ is a free parameter; hereafter we take $N_{N}=3$, which is the minimal number needed to get dark matter and the observed neutrino properties, through consistent assumptions 
about the flavor structure of the neutrino sector that will be explained presently. The HNLs couple to the SM lepton doublets as

$$
\eta_{\nu, i j} \bar{N}_{R, i} H L_{j}
$$

At energy scales relevant for inflation and below, it is consistent to assume that the only source of lepton number violation is through a small Majorana mass $\epsilon_{\nu}$ for the standard model neutrinos, which could be generated through the seesaw mechanism, by integrating out very heavy right-handed neutrinos, with mass $M_{\nu_{R}}$ above the scale of inflation. In the basis $\nu_{L}, N_{R}^{c}, N_{L}$, the neutrino mass matrix is

$$
\left(\begin{array}{ccc}
\epsilon_{\nu} & \eta_{\nu}^{T} \bar{v} & 0 \\
\eta_{\nu} \bar{v} & 0 & M_{N} \\
0 & M_{N} & 0
\end{array}\right)
$$

where $\bar{v} \cong 174 \mathrm{GeV}$ is the complex Higgs VEV. We assume that $\epsilon_{\nu}$ has a flavor structure that is aligned with the couplings in (2.2) as

$$
\epsilon_{\nu}=\bar{\mu}_{\nu} \eta_{\nu}^{T} \eta_{\nu}
$$

where $\bar{\mu}_{\nu}$ is a scale that we will constrain below. This alignment ensures the stability of dark matter against oscillations with its antiparticle, if $\eta_{\nu}$ has one vanishing eigenvalue. In order to justify the ansatz, we will show that it is radiatively stable, due to an approximate $\mathrm{SU}(3)$ flavor symmetry for the $N_{i}$ leptons, that is broken in a minimal-flavor-violating (MFV) [19] manner, solely by the matrix $\eta_{\nu}$. For example, the flavor-diagonal couplings of the inflaton to $N_{i}$ could be perturbed by a term proportional to $\eta_{\nu} \eta_{\nu}^{T}$ without spoiling the viability of the framework.

By solving for the eigenvalues of (2.3), one finds that the light neutrino part $\epsilon_{\nu}$ induces a small Majorana mass matrix for the $N_{i}$ 's of the form

$$
\delta M=\frac{\bar{v}^{2}}{M_{N}^{2}} \eta_{\nu} \epsilon_{\nu} \eta_{\nu}^{T}
$$

that leads to $N_{i}-\bar{N}_{i}$ oscillations. These are mildly constrained by the need for approximate lepton number conservation during the generation of the lepton asymmetry (apart from electroweak sphalerons), as we consider below.

\section{Nonstandard leptogenesis and DM relic density}

During inflation $\phi$ gets an asymmetry determined mostly by the couplings in eq. (1.1) and to a smaller extent by the initial conditions of the inflaton, which provide the source of $\mathrm{CP}$ violation in the Affleck-Dine mechanism [11]. The details of asymmetry generation at the level of $\phi$ are exactly the same as discussed in ref. [12]. The difference in the present work is that the $\phi$ asymmetry is transferred to the HNLs by the decays $\phi \rightarrow N N$ from the interaction (2.1). Whether reheating is perturbative or proceeds by parametric resonance is not crucial to the present discussion, where we assume that the created asymmetry results 
in the observed baryon asymmetry. This can always be achieved by appropriate choice of the $L$-violating parameter $\lambda^{\prime}$, for example. ${ }^{2}$

\subsection{Sharing and preserving the asymmetry}

For simplicity, consider the case where $g_{\phi}$ is sufficiently small so that perturbative decays are the dominant mechanism for reheating, with reheat temperature of order

$$
T_{R} \sim g_{\phi}\left(m_{\phi} m_{P}\right)^{1 / 2} \sim 10^{-3} g_{\phi} m_{P}
$$

using the typical value $m_{\phi} \sim 10^{-6} m_{P}$ identified in ref. [12]. Even for rather small values $g_{\phi} \lesssim 0.01$, this is well above the weak scale. Therefore it is easy for the HNLs to equilibrate with the SM through the interaction (2.2), which transmits the primordial $B-L$ asymmetry to the SM. The dominant process is $N_{i}$ (inverse) decays, whose rate is $\Gamma_{d} \cong 10^{-3} \eta_{\nu}^{2} T$ [21] for $T \gtrsim 100 \mathrm{GeV}$. Demanding that this comes into equilibrium before sphalerons freeze out, we find the lower bound $\left|\eta_{\nu}\right| \gtrsim 4 \times 10^{-7}$ on the largest elements of $\eta_{\nu, i j}$.

We demand that no $L$-violating effects from the operator $\lambda^{\prime} \phi^{4}$ in eq. (1.1) ever come into equilibrium, since these would wash out the asymmetry. Above the scale $m_{\phi}$, this comes from $\phi \phi \rightarrow \phi^{*} \phi^{*}$ scatterings with rate $\sim \lambda^{\prime 2} T$, that comes into equilibrium at $T \sim \lambda^{\prime 2} m_{P} \sim 10^{-24} m_{P}$, using the typical value $\lambda^{\prime} \sim 10^{-12}$ found in ref. [12]. This is far below $m_{\phi}$, hence it never comes into equilibrium. Instead the principal effect of $\lambda^{\prime}$ is through the effective operator $\left(\lambda^{\prime} g_{\phi}^{4} / m_{\phi}^{8}\right)\left(\bar{N} N^{c}\right)^{4}$ generated by integrating out the inflaton. This has a rate going as $\lambda^{\prime 2} g_{\phi}^{8} m_{\phi}^{-16} T^{17}$, that goes out of equilibrium at $T \sim\left[m_{\phi}^{16} /\left(\lambda^{\prime 2} g_{\phi}^{8} m_{p}\right)\right]^{1 / 15}$. Demanding that this remains below the reheat temperature gives an upper bound on $g_{\phi}$,

$$
g_{\phi} \lesssim\left(\frac{m_{\phi}}{m_{P}}\right)^{17 / 23}\left(\frac{1}{\lambda^{\prime}}\right)^{2 / 23} \cong 0.07
$$

which is not prohibitive.

The only other $L$-violating process operative at scales below that of inflation is $N$ $\bar{N}$ oscillations induced by the $\delta M$ matrix elements (2.5). These would wash out the $B$ and $L$ asymmetries if they were in equilibrium before sphaleron freezeout. The rate of $L$ violation is not simply the same as the oscillation rate $\sim 1 / \delta M$, because flavor-nondiagonal interactions of $N$ with the plasma can measure the state of the oscillating $N-\bar{N}$ system before it has time to oscillate significantly, damping the conversions of $N \rightarrow \bar{N}$. The effective rate of $L$ violation can be parametrized as $[22,23]$

$$
\Gamma_{\Delta L} \sim \frac{M_{N}^{2} \delta M^{2}}{M_{N}^{2} \delta M^{2}+T^{2} \Gamma_{m}^{2}} \Gamma_{m}
$$

where $\Gamma_{m}$ is the rate of processes that destroy the coherence of the $N-\bar{N}$ system. ${ }^{3}$ For $T>T_{\mathrm{EW}} \sim 100 \mathrm{GeV}$, (inverse) decays are dominant, but these quickly go out of equilibrium

\footnotetext{
${ }^{2}$ This observation is consistent with the results obtained by including the effects from nonlinear preheating dynamics on the generation of matter-antimatter asymmetry in Affleck-Dine inflationary scenarios [20].

${ }^{3}$ We will introduce an additional elastic scattering channel mediated by a singlet scalar $s$ below. These flavor-conserving interactions are not relevant for decohering the $N-\bar{N}$ oscillations [24].
} 
as $T$ falls below the mass of the Higgs boson. At temperatures somewhat below $T_{\mathrm{EW}}$, the elastic (but flavor-violating) $N L \rightarrow \bar{N} L$ scatterings mediated by Higgs exchange dominate, with $\Gamma_{m}=\Gamma_{\mathrm{el}} \sim \eta_{\nu}^{4} T^{5} / m_{h}^{4}$. On the other hand, sphalerons are safely out of equilibrium since they are exponentially suppressed by the Boltzmann factor involving the sphaleron energy, which is above the $\mathrm{TeV}$ scale. Therefore it is sufficient to show that the rate (3.3) is out of equilibrium in this case, to establish that the washout process is innocuous. In other words, the following relation must be satisfied

$$
\frac{\Gamma_{\Delta L}}{H} \sim \frac{M_{N}^{2} \delta M^{2} m_{h}^{4} m_{P}}{\eta_{\nu}^{4} T_{\mathrm{EW}}^{9}}<1
$$

In section 4 we will show that the light neutrino mass matrix $m_{\nu}$ is approximately equal to $\epsilon_{\nu}$, which is generated by integrating out heavy neutrinos though the usual seesaw mechanism. This allows us to rewrite the HNL Majorana mass matrix $\delta M$ in eq. (2.5) as $\delta M \sim \bar{v}^{2} \eta_{\nu}^{2} m_{\nu} / M_{N}^{2} \sim U_{\ell i}^{2} m_{\nu}$ where $U_{\ell i}$ is the mixing angle between HNLs and light neutrinos. Plugging the latter in eq. (3.4), the $\eta_{\nu}$-dependence disappears and we can get a lower bound on the HNL Dirac mass, $M_{N} \gtrsim 4 \mathrm{MeV}$. For higher values of $M_{N}$, the leptonviolating effects of $\delta M$ are therefore too small to affect the baryon asymmetry, but they can be observable in collider experiments that we will discuss in section 4 .

DM-antiDM oscillations for asymmetric DM have been considered in refs. [24, 25]. They can potentially regenerate the symmetric component of the DM and lead to its dilution through annihilations. We avoid these constraints by the relation (2.5) that causes $\delta M$ to vanish when acting on the $N^{\prime}$ DM state.

\subsection{DM asymmetric abundance and maximum mass}

The relic density for fully asymmetric DM is determined by its chemical potential, which in our framework is related to the baryon asymmetry in a deterministic way, since the DM initially has the same asymmetry as the remaining two HNLs. The relation between the $\mathrm{DM}$ and baryon asymmetries can be found by solving the system of equilibrium constraints, similarly to ref. [26]. We generalize their network to include the extra HNL species, that satisfy the equilibrium condition

$$
\mu_{N}=\mu_{h}+\mu_{L}
$$

from the $\eta_{\nu}$ interactions. Eq. (3.5) only applies to the unstable HNL species since $N^{\prime}$ is conserved, and its chemical potential is fixed by the initial lepton asymmetry

$$
\mu_{N^{\prime}}=\frac{1}{6} L_{0}
$$

The factor of 6 comes from having three HNL species, each with two chiralities. We recall that $L_{0}$ is determined by the inflationary dynamics, and is especially sensitive to the value of the coupling $\lambda^{\prime}$. It is assumed that $\lambda^{\prime}$ has been adjusted so that $L_{0}$ takes the value needed to yield the observed baryon asymmetry, which we relate to $L_{0}$ in the following. 
Repeating the analysis of [26] we find the following equilibrium relations (setting the $W$ boson potential $\mu_{W}=0$ since $\left.T>T_{\mathrm{EW}}\right)$ :

$$
\begin{aligned}
L & =\frac{13}{3} \mu_{\nu}+\mu_{h}+2 \mu_{N^{\prime}} \\
& =\frac{95}{21} \mu_{\nu}+2 \mu_{N^{\prime}} \\
B & =-\frac{4}{3} \mu_{\nu} \\
\mu_{u_{L}} & =-\frac{1}{9} \mu_{\nu}, \quad \mu_{h}=\frac{4}{21} \mu_{\nu} \\
\mu_{N} & =\frac{11}{21} \mu_{\nu}
\end{aligned}
$$

where $L, B$ are the respective total chemical potentials for lepton and baryon number, $\mu_{\nu}$ is the sum of light neutrino chemical potentials, and $\mu_{h}$ is that of the Higgs. Since $B-L$ is conserved by sphalerons, we can relate these to the initial lepton asymmetry $L_{0}=6 \mu_{N^{\prime}}=(L-B): \mu_{\nu}=\frac{84}{123} \mu_{N^{\prime}}, B=-\frac{112}{123} \mu_{N^{\prime}}$. This allows us to determine the maximum mass of $N^{\prime}$ that gives the observed relic density:

$$
m_{N^{\prime}}=M_{N} \leq\left|\frac{B}{\mu_{N^{\prime}}}\right| \frac{\Omega_{c}}{\Omega_{b}} m_{n}=4.5 \mathrm{GeV}
$$

using the values $\Omega_{c}=0.265$ and $\Omega_{b}=0.0493$ from ref. [27] and the nucleon mass $m_{n}$.

The inequality (3.8) is only saturated if the symmetric DM component is suppressed to a negligible level. Otherwise a smaller value of $m_{N^{\prime}}$ is needed to compensate the presence of the symmetric component. We turn to the general case next.

\subsection{Dark matter annihilation and relic density}

In order to reduce the symmetric component of the DM to avoid overclosure of the universe, an additional annihilation channel is needed. The $t$-channel Higgs-mediated annihilations $N^{\prime} \bar{N}^{\prime} \rightarrow L \bar{L}$ are not strong enough, leading to $\langle\sigma v\rangle \lesssim 10^{-32} \mathrm{~cm}^{3} / \mathrm{s}$, in light of the bound $\left|\eta_{\nu}\right| \lesssim 10^{-3}$ to be derived in section 4 below. We need an additional particle with sufficiently strong couplings to the DM.

The simplest possibility is to introduce a singlet scalar $s$, with interactions

$$
g_{s} s \bar{N}_{i} N_{i}+\frac{1}{4} \lambda_{s}\left(s^{2}-v_{s}^{2}\right)^{2}+\lambda_{h s} h^{2} s^{2}
$$

that at tree level are diagonal in the $N_{i}$ flavors, and lead to mixing of $s$ with the Higgs $h$. We will consider two cases: (i) $m_{s}<m_{N^{\prime}}$ so that $N^{\prime} \bar{N}^{\prime} \rightarrow s s$ is allowed; (ii) $m_{s} \gtrsim 2 m_{N^{\prime}}$ so that there can be mild resonant enhancement of the $s$-channel cross section for $N^{\prime} \bar{N}^{\prime}$ annihilation to standard model particles, through the mixing of $s$ with the Higgs boson. For the nonresonant case, the $s$-channel amplitude for $N^{\prime} \bar{N}^{\prime} \rightarrow f \bar{f}$, where $f$ is the most strongly coupled kinematically accessible final state, is of the same order of magnitude as that for $N^{\prime}$-nucleon scattering, which is strongly constrained by direct detection (section 6), making this contribution too small to be sufficient for annihilation. We will see that this limitation can be overcome by resonant enhancement without requiring too much fine tuning of masses. 


\subsection{1 $\quad N^{\prime} \bar{N}^{\prime} \rightarrow s s$ annihilation}

We first consider the case when $m_{s}<m_{N^{\prime}}$. The cross section for $N^{\prime} \bar{N}^{\prime} \rightarrow s s$ is $p$-wave suppressed. Parameterizing the Mandelstam variable as $s=4 m_{N^{\prime}}^{2}(1+\epsilon)$ we find in the limit $m_{s} \ll m_{N^{\prime}}$ and $\lambda_{s} \ll g_{s}$ that

$$
\sigma \cong \frac{3 g_{s}^{4}}{64 \pi m_{N^{\prime}}^{2}} \frac{\epsilon^{1 / 2}}{(1+\epsilon)^{2}}
$$

(this is an analytic approximation to the exact result, which is more complicated). Carrying out the thermal average [28] with $x \equiv m_{N^{\prime}} / T$ gives

$$
\begin{aligned}
\langle\sigma v\rangle & \cong \frac{3 g_{s}^{4}}{16 \pi m_{N^{\prime}}^{2}} F(x) \\
F(x) & =\frac{x}{K_{2}(x)^{2}} \int_{0}^{\infty} d \epsilon\left(\frac{\epsilon}{1+\epsilon}\right)^{3 / 2} K_{1}(2 x \sqrt{1+\epsilon}) \\
& \cong 0.058-0.002 x+3.25 \times 10^{-5} x^{2}-1.87 \times 10^{-7} x^{3}
\end{aligned}
$$

which is a good numerical approximation in the region $15<x<70$. For values $x \sim 20$ typical for freezeout, $F \cong 0.03$.

To find the relic abundance including both symmetric and asymmetric components, one can solve the Boltzmann equation for their ratio $r$ given in ref. [29], which depends upon $\langle\sigma v\rangle$. Then as shown there, the fractional contribution of $N^{\prime}$ to the energy density of the universe is

$$
\Omega_{N^{\prime}}=\epsilon \eta_{B} m_{N^{\prime}} \frac{s}{\rho_{\text {crit }}}\left(\frac{1+r}{1-r}\right)
$$

where $\eta_{B}=8.8 \times 10^{-11}$ is the observed baryon-to-entropy ratio, $s$ is the entropy density, and $\epsilon=\eta_{N^{\prime}} / \eta_{B}=123 / 112$ in our model (see below eq. (3.7)). Using ref. [30], we checked whether the DM annihilation cross section might be Sommerfeld-enhanced since $m_{s}<m_{N^{\prime}}$, but this was a negligible effect in the relevant parts of parameter space that we will specify below. In figure 1 (left) we plot contours of $\Omega_{N^{\prime}}$, the fractional contribution of the DM to the energy density of the universe, in the plane of $m_{N^{\prime}}$ versus $g_{s}$. For $g_{s} \gtrsim 0.14$ the maximum value in eq. (3.8) is achieved, whereas for lower $g_{s}$, the symmetric component abundance is increased (while the asymmetric abundance remains fixed), corresponding to lower DM masses.

In the opposite regime $\lambda_{s} \gg g_{s}$, the annihilation $N^{\prime} \bar{N}^{\prime} \rightarrow s s$ could in principle be dominated by the $s$-channel diagram, giving the cross section

$$
\langle\sigma v\rangle \cong \frac{1}{\pi}\left(\frac{3 \lambda_{s} v_{s} g_{s}}{8 m_{N^{\prime}}^{2}}\right)^{2} \bar{F}(x)
$$

in the case where $m_{s} \ll m_{N^{\prime}}$, with

$$
\bar{F}(x)=\frac{x}{K_{2}(x)^{2}} \int_{0}^{\infty} d \epsilon \frac{\epsilon^{3 / 2}}{(1+\epsilon)^{5 / 2}} K_{1}(2 x \sqrt{1+\epsilon})
$$



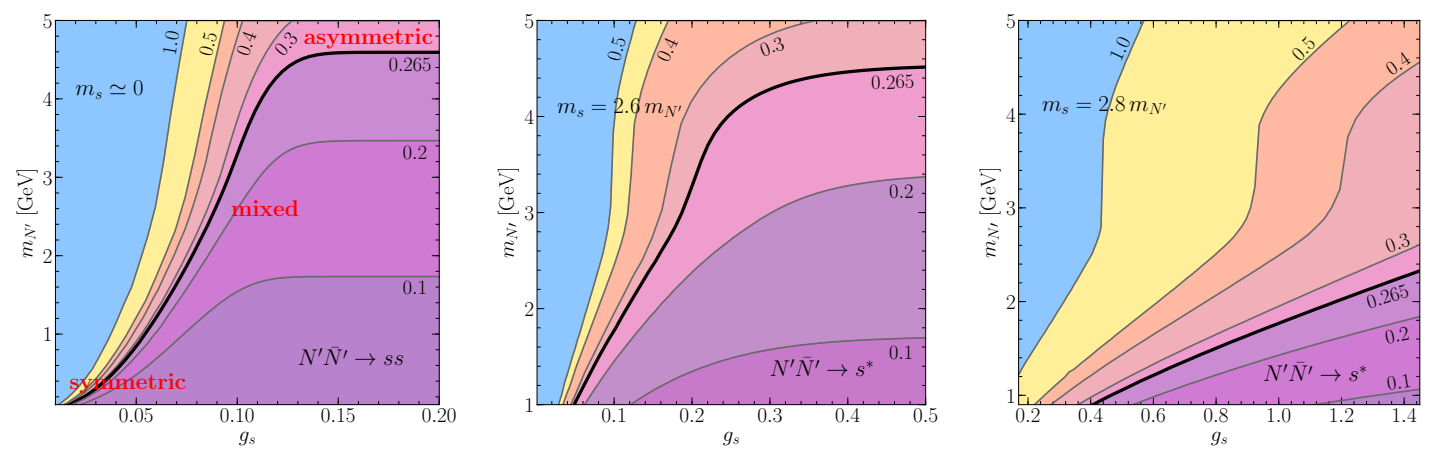

Figure 1. Contours of DM relic density $\Omega_{N^{\prime}} \equiv \rho_{N^{\prime}} / \rho_{\text {crit }}$ in the plane of DM mass versus coupling to singlet, for three relations of singlet mass $m_{s}$ to the DM mass $m_{N^{\prime}}$. Left: $m_{s} \ll m_{N^{\prime}}$, with $N^{\prime} \bar{N}^{\prime} \rightarrow s s$ annihilation. Center: $m_{s}=2.6 m_{N^{\prime}}$ with $N^{\prime} \bar{N}^{\prime} \rightarrow s^{*}$ (virtual $s$ ) annihilation. Right: like center, but with $m_{s}=2.8 m_{N^{\prime}}$ The heavy contour labeled 0.265 corresponds to the observed relic density.

For $x \sim 20, F \cong 0.01$ leading to the requirement that $g_{s}$ must be significantly larger than in the previous case to suppress the symmetric DM component. Such values are excluded by direct DM search constraints to be discussed in section 6 below. Hence there is no practical enlargement of the allowed parameter space from including the $s$-channel contribution.

\subsection{2 $\quad N^{\prime} \bar{N}^{\prime} \rightarrow$ SM annihilation}

In the other case where $m_{s}>m_{N^{\prime}}$, the total annihilation cross section for $N^{\prime} \bar{N}^{\prime}$ into $\mu^{+} \mu^{-}, \pi^{+} \pi^{-}$, etc., through the Higgs portal, does not depend upon the couplings of $s$ to the final state particles nor on the number of decay channels, in the limit of the narrowwidth approximation for the intermediate virtual $s$. In this limit we can approximate the Breit-Wigner distribution for the $s$ propagator as a $\delta$ function, $\left(\pi / \Gamma_{s}\right) \delta\left(s-m_{s}^{2}\right)[s$ is the Mandelstam variable], and the couplings in the singlet decay width $\Gamma_{s}$ cancel against those in the annihilation amplitude. One can think of this as the cross section for $N^{\prime} \bar{N}^{\prime} \rightarrow s$, which one integrates over the $\delta$ function when doing the thermal average. In this way we find

$$
\langle\sigma v\rangle \cong \pi \frac{g_{s}^{2}}{2 m_{N^{\prime}}^{2}} \frac{\left(y^{2}-1\right)^{3 / 2} x}{K_{2}(x)^{2}} K_{1}(2 x y)
$$

where $x=m_{N^{\prime}} / T$ as usual, and $y \equiv m_{s} /\left(2 m_{N^{\prime}}\right)$. It turns on steeply above the threshold $y=1$ for resonant enhancement, and then quickly decays because of the Boltzmann suppression for $y \gg 1$. Nevertheless we find that it can be large enough for values of $y \lesssim 1.3-1.4$ that are not finely tuned to be close to 1 , as we show in figure 1 (center and right plots).

We will see that for such parameter values, the $t$-channel exchange of $s$ for $N^{\prime}$ scattering on nucleons can still be consistent with direct detection constraints. In this process, the suppression by the small coupling of $s$ to nucleons (through the singlet-Higgs mixing angle $\theta_{s}$ ) is not canceled by anything, in contrast to the $s$-channel resonance. 


\section{Neutrino properties and HNL constraints}

Below the scales of electroweak symmetry breaking and the HNL mass $M_{N}$, the light neutrino mass matrix gets generated,

$$
\begin{aligned}
m_{\nu} & \cong \epsilon_{\nu}-\delta M^{\prime} \\
\delta M^{\prime} & \equiv \frac{\bar{v}^{2}}{M_{N}^{2}} \eta_{\nu}^{T} \epsilon_{\nu} \eta_{\nu}
\end{aligned}
$$

However $\left|\eta_{\nu}\right| \bar{v} / M_{N} \ll 1$ is the magnitude of the mixing between the light neutrinos and the HNLs, as we will discuss below, so that the correction $\delta M^{\prime} \ll \epsilon_{\nu}$ can be ignored. We reiterate that $\epsilon_{\nu}$ is generated by the usual seesaw mechanism, integrating out sterile neutrinos whose mass is above all the other relevant scales in our model.

Recall that the stability of the dark matter $N^{\prime}$ requires $\eta_{\nu}$ to be a matrix with one vanishing eigenvalue, which implies that the lightest neutrino is massless. This is an exact statement, not relying upon the neglect of $\delta M^{\prime}$, since $\epsilon_{\nu}$ and $\delta M^{\prime}$ are simultaneously diagonalizable by construction. This is a consequence of our MFV-like assumption that $\eta_{\nu}$ is the only source of flavor-breaking in the HNL/neutrino sector.

\subsection{Explicit $\eta_{\nu}$ and HNL mixings}

Using eq. (2.4) we can solve for $\eta_{\nu}$ in terms of the neutrino masses and mixings,

$$
\eta_{\nu}=O\left(\frac{D_{\nu}}{\bar{\mu}_{\nu}}\right)^{1 / 2} U_{\mathrm{PMNS}}^{-1}
$$

where $D_{\nu}$ is the diagonal matrix of light $\nu$ mass eigenvalues, and $U_{\mathrm{PMNS}}$ is the $3 \times 3$ PMNS matrix. The orthogonal matrix $O$ is undetermined since the $N_{i}$ are practically degenerate; for simplicity we set it to 1 in the following. Since we have assumed that one eigenvalue is vanishing, the other two are known,

$$
\begin{array}{ll}
D_{\nu 11}=0, D_{\nu 22}=\sqrt{\Delta m_{21}^{2}}, \quad D_{\nu 33}=\sqrt{\Delta m_{31}^{2}}, & \mathrm{NH} \\
D_{\nu 33}=0, D_{\nu 22}=\sqrt{\Delta m_{32}^{2}}, \quad D_{\nu 11}=\sqrt{\Delta m_{32}^{2}-\Delta m_{21}^{2}}, & \mathrm{IH}
\end{array}
$$

for the normal and inverted hierarchies, respectively.

The light neutrinos mix with $N_{i}$, with mixing matrix elements given by

$$
U_{\ell i} \cong \frac{\eta_{\nu, \ell i}^{T} \bar{v}}{M_{N}}
$$

where $\ell=e, \mu, \tau$ and $i=1,2,3$. Constraints on $U_{\ell i}$ arise from a variety of beam dump experiments and rare decay searches, summarized in refs. [14, 31]. As we now discuss, the applicability of these limits depends upon whether the scalar singlet is heavier or lighter than the HNL's, since this determines the dominant decay modes of the latter. 


\subsubsection{Unitarity constraints for $m_{s}<M_{N}$ case}

If $m_{s}<M_{N}$, then many of the beam-dump and other limits on the mixing angles (4.4) versus $m_{N}$, shown in figure 2 , cannot be directly applied to our model because they assume that $N$ decays are mediated only by the weak interactions, through $N-\nu$ mixing, whereas we have a more efficient decay channel $N \rightarrow \nu s$, from the $g_{s} \bar{N}_{i} N_{i}$ coupling and mixing. All of the bounds that rely upon detecting visible particles from the decay will now be sensitive to the singlet mass $m_{s}$ and mixing angle $\theta_{s}$ with the Higgs, and not just $M_{N}$. To modify these limits appropriately would require a dedicated reanalysis of each experiment, which is beyond the scope of our work.

However we can still make a definite statement about how weak the limit on $N-\nu$ mixing could possibly be, even in the case where the singlet escapes the detector unobserved, because electroweak precision data (EWPD) are only sensitive to the reduction in the SM couplings caused by the mixing, that we can readily calculate. This is most straightforward in the basis of the mass eigenstates, where $\eta_{\nu}$ is diagonal. Then the mass matrix (2.3) is block diagonal, and there is a mixing angle $\theta_{i}$ connecting each pair of light and heavy mass eigenstates. The relation between the flavor states (labeled by subscript $\alpha$ ) and the mass eigentstates (labeled by $i$ ) is

$$
\nu_{\alpha}=\left(U_{\mathrm{PMNS}}\right)_{\alpha i} \cos \theta_{i} \nu_{i} \equiv N_{\alpha i} \nu_{i}
$$

In refs. [32, 33], the matrix $N_{\alpha i}$ is introduced in this way to parametrize departures from unitarity in the lepton mixing matrix, and the magnitudes of $N N^{\dagger}$ are constrained by various precision electroweak data. The elements of such a matrix can be written in our model as

$$
\left|N N^{\dagger}\right|_{\alpha \beta} \equiv\left|\sum_{i} N_{\alpha i} N_{i \beta}^{\dagger}\right|=\left|\delta_{\alpha \beta}-\sum_{i}\left(U_{\mathrm{PMNS}}\right)_{\alpha i} \sin ^{2} \theta_{i}\left(U_{\mathrm{PMNS}}\right)_{i \beta}^{\dagger}\right|
$$

Since most of the constraints on physical observables are often expressed in literature in terms of the Hermitian matrix $\varepsilon_{\alpha \beta}$, defined in $N=(1-\varepsilon) U_{\text {PMNS }}$ [33], we have that the predicted $\varepsilon_{\alpha \beta}$ turns out to be ${ }^{4}$

$$
\varepsilon_{\alpha \beta}=\frac{1}{2}\left|\sum_{i}\left(U_{\mathrm{PMNS}}\right)_{\alpha i} \sin ^{2} \theta_{i}\left(U_{\mathrm{PMNS}}\right)_{i \beta}^{\dagger}\right|
$$

The most stringent limits on $\varepsilon_{\alpha \beta}$ that can be applied to our model come from the measurement of the $W$ boson mass, which depends upon the combination [33]

$$
\begin{aligned}
M_{W} & \simeq M_{W}^{\mathrm{SM}}\left[\left(N N^{\dagger}\right)_{e e}\left(N N^{\dagger}\right)_{\mu \mu}\right]^{1 / 4} \frac{s_{W}^{\mathrm{SM}}}{s_{W}} \\
& \cong M_{W}^{\mathrm{SM}}\left(1+0.20\left(\varepsilon_{e e}+\varepsilon_{\mu \mu}\right)\right)
\end{aligned}
$$

where the SM radiative corrections, parametrized by the variable $\Delta r=0.03672$ [34], are included in the computation; they enter through the weak mixing angle [32],

$$
s_{W}^{2}=\frac{1}{2}\left[1-\sqrt{1-\frac{2 \sqrt{2} \pi \alpha}{G_{\mu} M_{Z}^{2}}(1+\Delta r)\left[\left(N N^{\dagger}\right)_{e e}\left(N N^{\dagger}\right)_{\mu \mu}\right]^{1 / 2}}\right]
$$

\footnotetext{
${ }^{4}$ The matrix $\varepsilon$ defined here is called $\eta$ in ref. [33].
} 
Using the experimental and SM values of $M_{W}$ in eq. (4.8), we obtain a 95\% C.L. upper bound on $\left(\varepsilon_{e e}+\varepsilon_{\mu \mu}\right) \leq 2.64 \times 10^{-3}$.

In our framework, the mixing angles $\theta_{i}$ in eq. (4.7) and be computed explicitly, from the eigenvalues of $\eta_{\nu}$, up to multiplicative factors,

$$
\theta_{i} \cong \frac{\eta_{i} \bar{v}}{M_{N}}
$$

where $\eta_{i}$ is the eigenvalue of $\eta_{\nu}$ associated with the eigenvector that couples to $N_{i}$. For the normal hierarchy, we label $\eta_{1}=0$ for the massless state, while for inverted hierarchy $\eta_{3}=0$. Using (4.2), we can solve explicitly for $\eta_{\nu}$ in either mass scheme, up to the overall proportionality controlled by the parameter $\bar{\mu}_{\nu}$. Comparing the combination $\left(\varepsilon_{e e}+\varepsilon_{\mu \mu}\right)$, computed from eqs. (4.7) and (4.10), to the upper limit found above from $M_{W}$, yields lower bounds on the scale $\bar{\mu}_{\nu}$ in the two mass hierarchy choices, and upper bounds on the corresponding matrices $\eta_{\nu}$ and the mixing angles between HNLs and the light neutrinos. Defining $\bar{U}_{\ell} \equiv\left(\sum_{i}\left|U_{\ell i}\right|^{2}\right)^{1 / 2}$, we find for the normal mass hierarchy

$$
\begin{gathered}
\bar{\mu}_{\nu}>5.9 \mathrm{keV} \times\left(\frac{4.5 \mathrm{GeV}}{M_{N}}\right)^{2}, \\
\left|\eta_{\nu}^{T}\right|<10^{-3}\left|\begin{array}{ccc}
0 & 0.66 & -0.32-0.29 i \\
0 & 0.72-0.05 i & 2.14 \\
0 & -0.70-0.04 i & 1.91
\end{array}\right| \times\left(\frac{M_{N}}{4.5 \mathrm{GeV}}\right) \\
\bar{U}_{e}<0.031, \quad \bar{U}_{\mu}<0.087, \quad \bar{U}_{\tau}<0.078
\end{gathered}
$$

The dependence on $M_{N}$ cancels out in the bounds on $U_{\ell i}$. The corresponding results for inverted hierarchy are

$$
\begin{array}{cc}
\bar{\mu}_{\nu}>13.6 \mathrm{keV} \times\left(\frac{4.5 \mathrm{GeV}}{M_{N}}\right)^{2}, & \mathrm{IH} \\
\left|\eta_{\nu}^{T}\right|<10^{-3}\left|\begin{array}{ccc}
1.57 & 1.06 & 0 \\
-0.75-0.17 i & 1.02-0.12 i & 0 \\
0.75-0.15 i & -1.23-0.10 i & 0
\end{array}\right| \times\left(\frac{M_{N}}{4.5 \mathrm{GeV}}\right) \\
\bar{U}_{e}<0.073, \quad \bar{U}_{\mu}<0.050, \quad \bar{U}_{\tau}<0.056
\end{array}
$$

In each case the column of zeros corresponds to the absence of coupling to the DM state $N^{\prime}$; hence we identify $N^{\prime}=N_{1}$ for the normal hierarchy and $N^{\prime}=N_{3}$ for the inverted hierarchy.

We emphasize that the above bounds are robust, but might be strengthened, depending on the choices of $m_{s}$ and $\theta_{s}$, by reanalyzing limits from other experiments to take into account the observation of charged particles or neutral hadrons from $s$ decays following $N \rightarrow \nu s$. Hence the true limits are expected to lie somewhere between the (brown) EWPD line shown in figure 2 for the normal (left) and inverted (right) hierarchy cases, and the more stringent limits that may arise from the other (typically beam dump) experiments. 

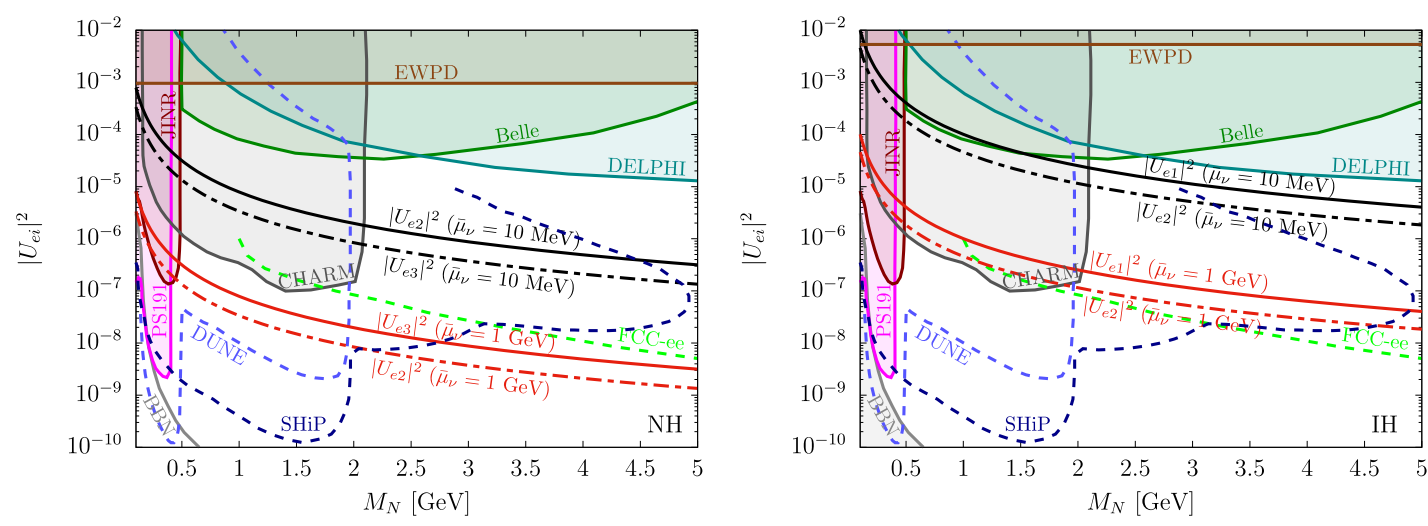

Figure 2. Summary of constraints on HNL mixing with electron neutrinos, over mass range of interest for our model (left: normal hierarchy, right: inverted hierarchy). Solid and dot-dashed black and red curves show the model's predictions for $U_{e 2}\left(U_{e 1}\right)$ (solid curves) and $U_{e 3}\left(U_{e 2}\right)$ (dot-dashed) in the normal (inverted) mass hierarchy, for two choices of the parameter $\bar{\mu}_{\nu}$ that determines the mixing through eqs. (4.2), (4.4). $U_{e 1} \equiv 0\left(U_{e 3} \equiv 0\right)$ for the normal (inverted) hierarchy since $N_{1}=N^{\prime}\left(N_{3}=N^{\prime}\right)$ denotes the dark matter HNL. Laboratory constraints are taken from ref. [14]. Although a more recent and comprehensive analysis of these bounds in the $\mathrm{MeV}-\mathrm{GeV}$ mass range was made in ref. [35], we noticed no appreciable difference for $\mathrm{MN}>0.1 \mathrm{GeV}$. We also do not display the preliminary limit from the NA62 experiment [36], which would be the strongest limit for MN between 0.15 and $0.45 \mathrm{GeV}$ if confirmed. Sensitivity regions of future experiments FCC-ee [37], DUNE [38] and SHiP [39] are bounded by dashed curves.

The scale $\bar{\mu}_{\nu}$ determines how the couplings $y_{\nu}=k \eta_{\nu}$ of the light neutrinos to the superheavy Majorana neutrinos $\nu_{R}$ (as restricted by our MFV-like assumption) are enhanced relative to $\eta_{\nu}$ by a proportionality factor, $k=\left(M_{\nu_{R}} \bar{\mu}_{\nu} / \bar{v}^{2}\right)^{1 / 2}$. Perturbativity of $y_{\nu}$ limits $k \lesssim 0.5 \times 10^{3}$, hence the scale of the heavy neutrinos is bounded by $M_{\nu_{R}} \lesssim 10^{15} \mathrm{GeV}$ for the value of $\bar{\mu}_{\nu}$ in eq. (4.11). This is not restrictive, and can be made consistent with our assumption that the heavy neutrinos do not play a role during inflation or reheating, if the reheat temperature is sufficiently low.

\subsubsection{Laboratory constraints for $m_{s}>M_{N}$ case}

If $m_{s}>M_{N}$, only three-body decays of HNL's are available, and they are dominated by weak interactions, induced by mixing of $N_{i}$ with the light $\nu$ 's. There is also a 3-body decay $N \rightarrow \nu f \bar{f}$ by virtual $s$ exchange, but this is highly suppressed by the small scalar mixing angle $\theta_{s}$ and the couplings $m_{f} / v$ to the Higgs. In this case, all of the constraints on $N-\nu$ mixing shown in figure 2 unambiguously apply. For masses $M_{N}>2 \mathrm{GeV}$, the most stringent limit comes from searches for $Z \rightarrow N \nu$ decays by the DELPHI Collaboration [40]. Defining again $\bar{U}_{\ell}=\left(\sum_{i}\left|U_{\ell i}\right|^{2}\right)^{1 / 2}$, at our largest allowed mass $M_{N}=4.5 \mathrm{GeV}$, the bound is

$$
\bar{U} \equiv\left(\sum_{\ell} \bar{U}_{\ell}^{2}\right)^{1 / 2}<0.0039,
$$

since DELPHI was sensitive to the total rate of $N_{i}$ production from $Z \rightarrow N_{i} \nu_{\ell}$ decays, times the total (semi)leptonic rate of $N_{i}$ decays. 

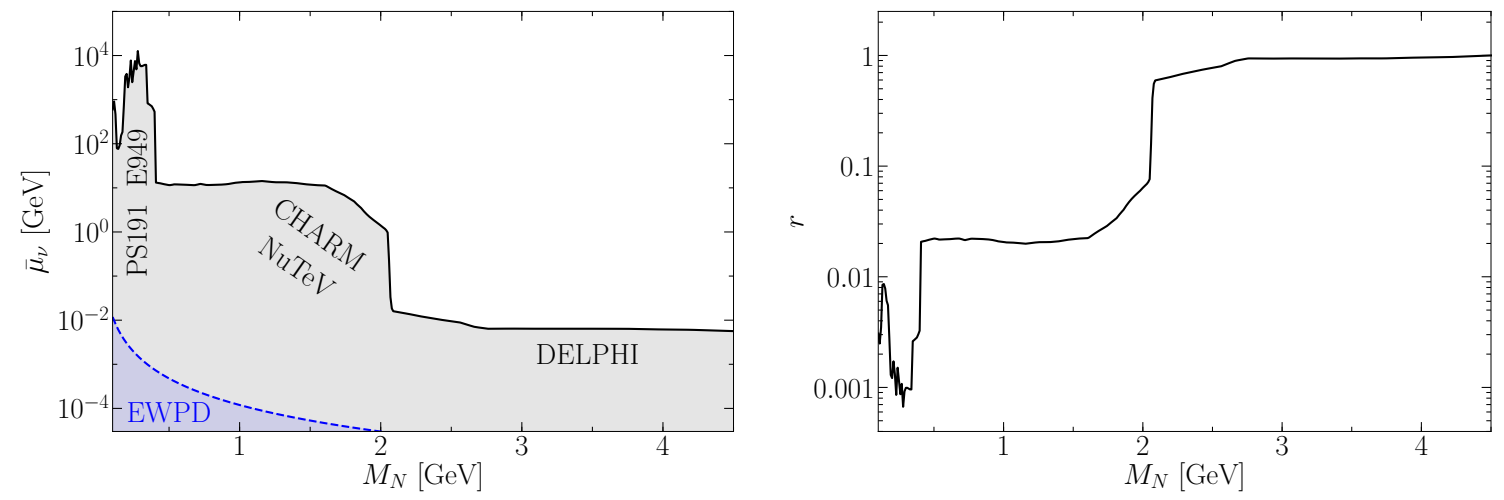

Figure 3. Left: minimum allowed mass scale $\bar{\mu}_{\nu}\left(M_{N}\right)$, predicted by our model for the normal mass hierarchy case, compatible with current constraints on the HNL mixings to light neutrinos [14]. The shaded gray region is excluded. Right: the ratio $r$ showing how the maximum allowed mixings (4.16) at $M_{N}=4.5 \mathrm{GeV}$ are rescaled at lower $M_{N}$.

Using eqs. (4.2), (4.4), the bound (4.13) can be approximately saturated if $\bar{\mu}_{\nu}=$ $5.7(9.8) \mathrm{MeV}$ for the normal (inverted) hierarchy. Taking the PDG central values of the neutrino masses and mixings [34], we find

$$
\begin{aligned}
& \eta_{\nu}^{T} \cong 10^{-5}\left(\begin{array}{ccc}
0 & 2.1 & -1.0-0.9 i \\
0 & 2.3-0.2 i & 6.9 \\
0 & -2.2-0.1 i & 6.1
\end{array}\right) \\
& \bar{U}_{e} \cong 0.00099, \quad \bar{U}_{\mu} \cong 0.0028, \quad \bar{U}_{\tau} \cong 0.0025
\end{aligned}
$$

at $M_{N}=4.5 \mathrm{GeV}$ for the normal hierarchy, and

$$
\begin{gathered}
\eta_{\nu}^{T} \cong 10^{-5}\left(\begin{array}{ccc}
5.8 & 3.9 & 0 \\
-2.8-0.6 i & 3.8-0.4 i & 0 \\
2.8-0.6 i & -4.6-0.4 i & 0
\end{array}\right) \\
\bar{U}_{e} \cong 0.0027, \quad \bar{U}_{\mu} \cong 0.0019, \quad \bar{U}_{\tau} \cong 0.0021
\end{gathered}
$$

for the inverted hierarchy. In each case the column of zeros corresponds to the absence of coupling to the DM state $N^{\prime}$; hence we identify $N^{\prime}=N_{1}$ for the normal hierarchy and $N^{\prime}=N_{3}$ for the inverted hierarchy.

For the lighter mass range $M_{N} \sim(0.4-2) \mathrm{GeV}$, beam dump experiments such as CHARM [41] and NuTEV [42] give the strongest limits for electron and muon flavors, roughly $U_{e i}, U_{\mu i} \lesssim 6 \times 10^{-4}\left(M_{N} / \mathrm{GeV}\right)^{-1.14}$. The largest allowed magnitudes of the HNL mixings $U_{\ell i}$ can be expressed as a function of $M_{N}$,

$$
\left|U_{\ell i}\right| \cong r\left(M_{N}\right)\left(\begin{array}{ccc}
0 & 0.00083 & 0.00054 \\
0 & 0.00090 & 0.0027 \\
0 & 0.00087 & 0.0024
\end{array}\right)
$$

focusing on the normal hierarchy case. We determined the minimum allowed value of $\bar{\mu}_{\nu}$ for lower $M_{N}$, and the consequent scaling factor $r\left(M_{N}\right)=\left(5.7 \mathrm{MeV} / \min \left(\bar{\mu}_{\nu}\right)\right)^{1 / 2}$, from the 
limits summarized in figures 4.10-4.12 of ref. [14]. These limits were rescaled and combined to account for the fact that our model has two HNLs, each of which mixes with all of the light flavors rather than just one $N_{i}$ that can mix with only one flavor at a time. The functions $\min \left(\bar{\mu}_{\nu}\right)$ and $r\left(M_{N}\right)$ are plotted in figure 3. The various constraints on the HNL mixing with electron neutrinos in the mass range relevant for our model are shown for two choices of $\bar{\mu}_{\nu}$ in figure 2, including future constraints from FCC-ee, DUNE and SHiP.

\section{$4.2 \quad N-\bar{N}$ oscillations}

As mentioned in section 3.1, the $L$-violating mass term $\delta M$ causes $N-\bar{N}$ oscillations at a rate that is too small to destroy the lepton asymmetry in the early universe, but fast enough to possibly be detected in laboratory searches. In the scenario where $m_{s}<M_{N}$, this effect cannot be observed because the decay products of $N_{i} \rightarrow \nu \mu^{+} \mu^{-}$and $\bar{N}_{i} \rightarrow \bar{\nu} \mu^{+} \mu^{-}$differ only by having a neutrino versus antineutrino in the final states. However if $m_{s}>M_{N}$, the situation is more interesting. For the values of $\bar{\mu}_{\nu}$ and $\eta_{\nu}$ in eq. (4.14), the largest eigenvalue of $\delta M$ is given by ${ }^{5}$

$$
\delta M=3.1 \times 10^{-6} \mathrm{eV}\left(\frac{2 \mathrm{GeV}}{M_{N}}\right)^{2},
$$

It was recently shown by ref. [43] that this is a promising value for inducing observable $N-\bar{N}$ oscillations at the SHiP experiment. These would be seen by production of $N \ell^{+}$in a hadronic collision, followed by semileptonic decays $N \rightarrow \bar{N} \rightarrow \ell^{+} \pi$ (where $\pi$ represents a generic hadron). The smoking gun is the presence of like-sign leptons in the decay chain, that can only occur if $N$ oscillates to $\bar{N}$ within the detector.

\subsection{Weak HNL decays}

In the case where $m_{s}>M_{N}$ so that $N_{i} \rightarrow \nu s$ decays are blocked, the lifetime of the unstable $N_{i}$ leptons is determined by weak decays. These can be 2- or 3-body, $N_{i} \rightarrow \ell^{-} q \bar{q}$ (with $q \bar{q}$ hadronizing into a meson) and $N_{i} \rightarrow \nu \ell^{+} \ell^{-}$by $W$ and $Z$ exchange, due to mixing of $N_{i}$ with the active neutrinos with mixing angles $U_{i \ell}^{T} \cong-U_{\ell i}$. Then the decay rate is of order

$$
\Gamma_{N_{i}} \sim \sum_{\ell} \frac{\left|U_{\ell i}\right|^{2} G_{F}^{2} M_{N}^{5}}{192 \pi^{3}}
$$

This gives a lifetime of $\sim 10^{-3}-10^{-4}$ s for $M_{N} \sim 1 \mathrm{GeV}$, making such $N_{i}$ decays harmless for big bang nucleosynthesis (BBN) or the CMB.

More quantitatively, we have evaluated the partial widths for $N_{i} \rightarrow \nu \gamma, N_{i} \rightarrow h^{0} \nu$, $N_{i} \rightarrow h^{+} \ell^{-}, N_{i} \rightarrow 3 \nu, N_{i} \rightarrow \nu \ell^{+} \ell^{-}$, including the hadronic final states with $h^{0}=$ $\pi^{0}, \eta, \eta^{\prime}, \rho^{0}, h^{+}=\pi^{+}, K^{+}, \rho^{+}, D^{+}$as computed in ref. [44] and [45]. ${ }^{6} \quad$ Focusing on the

\footnotetext{
${ }^{5}$ The eigenvalue of $\delta M$ computed in eq. (4.17) is the maximum value allowed by current experimental constraints because $\delta M \propto \bar{\mu}_{\nu}^{-1}$ from eqs. (2.5), (4.2) and the minimum of $\bar{\mu}_{\nu}$ is reached at $M_{N}=4.5 \mathrm{GeV}$ as shown in figure 3 .

${ }^{6}$ The formula for the decay width of $N_{i} \rightarrow \nu \ell_{1}^{+} \ell_{2}^{-}$found in the literature (see refs. [44, 46]) assumes that $m_{\ell_{1}}$ is negligible compared to $m_{\ell_{2}}$. This is not as good an approximation for the case $\ell_{1}=\mu, \ell_{2}=\tau$ as for $\ell_{1}=e, \ell_{2}=\mu$. We provide the exact formula in appendix A.
} 

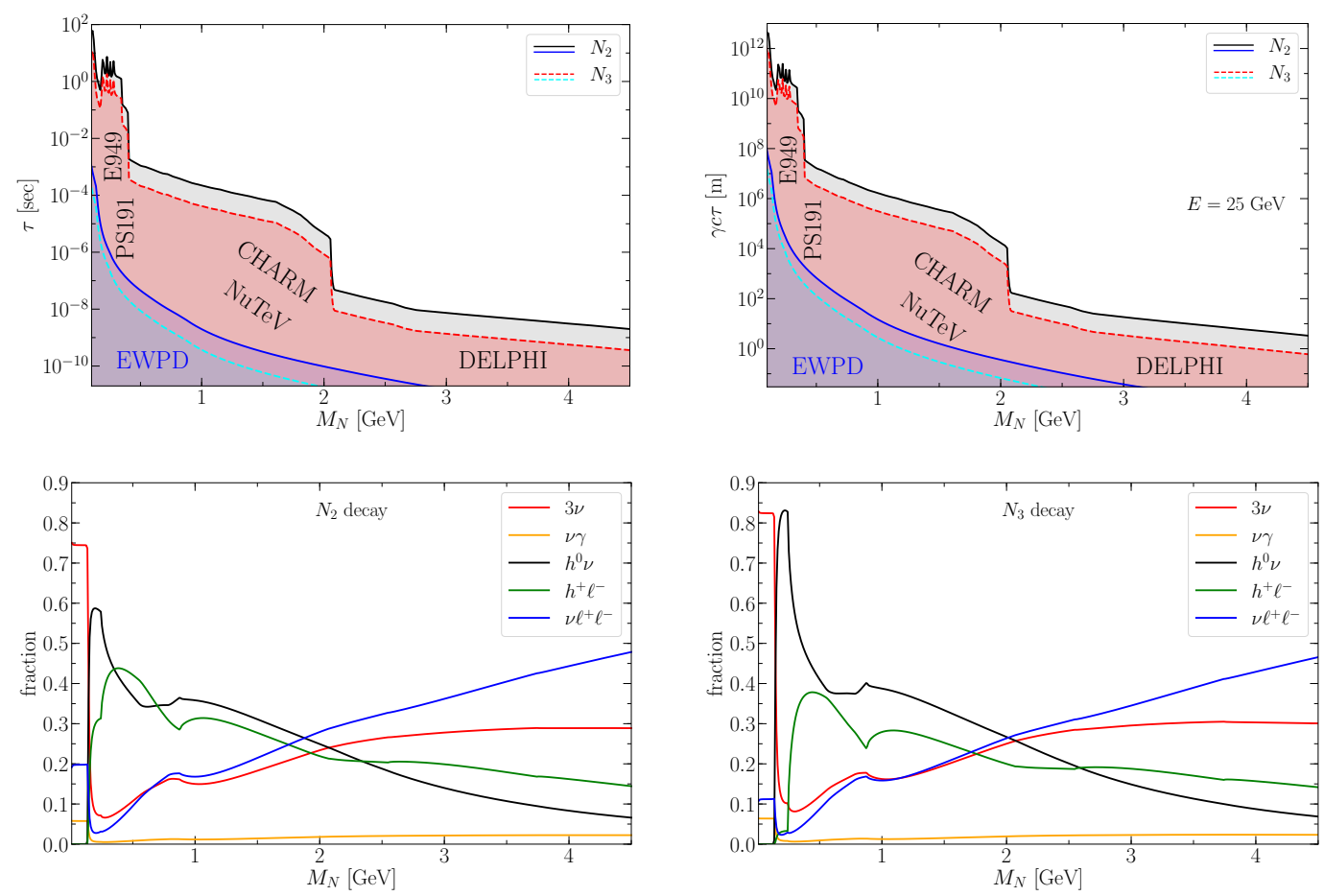

Figure 4. Top: minimum lifetime (left) and decay length (right) of the HNLs $N_{2}$ and $N_{3}$, for the case of normal mass hierarchy. Upper curves are for mass $M_{N}<m_{s}$, lower curves for $M_{N}>m_{s}$, which determines whether weak decays or $N \rightarrow \nu s$ dominates. Decay length assumes energy $E=25 \mathrm{GeV}$, appropriate for SHiP experiment. The shaded regions are excluded. (The wiggles in the mass range $0.2<M_{N}<0.4 \mathrm{GeV}$ come from the E949 bound [47] present in figure 4.11 of ref. [14], which also appear in figure 3.) Bottom: branching ratios for $N_{2}$ (left) and $N_{3}$ (right) into various final states involving photon, hadrons, light neutrinos or charged leptons, for the case of weak decays, namely $M_{N}<m_{s}$.

normal hierarchy case, we use the mixing matrix given by eq. (4.4) with $\bar{\mu}_{\nu}$ shown in figure 3, that leads to different lifetimes for the two unstable HNLs $N_{2}$ and $N_{3}$. The lifetimes are plotted in figure 4, along with decay lengths in the case of HNLs with energy $E=25 \mathrm{GeV}$ that would be relevant for the SHiP experiment. For $M_{N} \lesssim 0.3 \mathrm{GeV}$, the lifetimes start to exceed $1 \mathrm{~s}$, which for generic models of HNLs would come into conflict with nucleosynthesis. In our model, this need not be the case since the HNL abundance is suppressed by $N_{i} \bar{N}_{i} \rightarrow s s$ annihilations. Then it is the singlet that should decay before BBN, which generally occurs as long as $m_{s}>2 m_{e}$.

In figure 4 the branching ratios for $N_{2}$ and $N_{3}$ to decay into the various final states (summing over flavors within each category) is also shown. Leptonic final states dominate for $M_{N}>2 \mathrm{GeV}$, while hadronic ones dominate for lower $M_{N}$.

\subsection{Entropy and energy injection by late $N$ decays}

If the Dirac HNLs $N_{2}$ and $N_{3}$ dominate the energy density of the universe and are sufficiently long-lived, which may happen if the two-body decay $N_{2,3} \rightarrow \nu s$ is kinematically forbidden, a large amount of entropy may be injected by the decay of these particles af- 

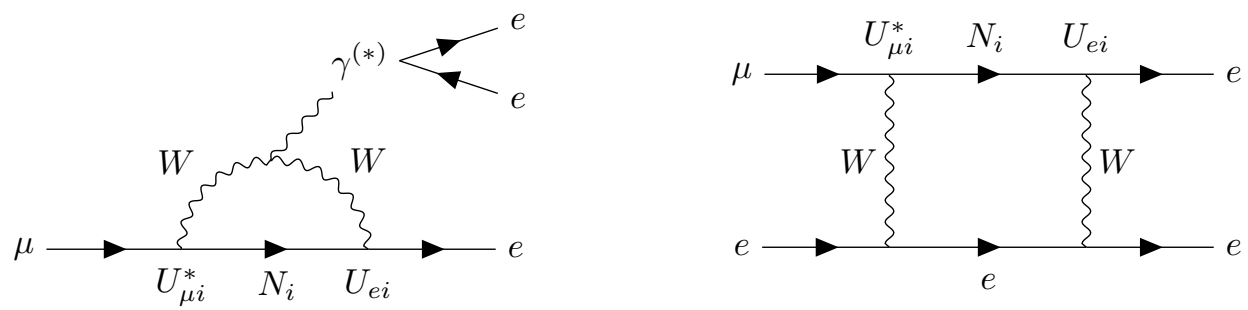

Figure 5. Diagrams leading to $\mu \rightarrow e \gamma$ and $\mu \rightarrow 3 e$ from mixing of HNL's with the light neutrinos.

ter freezeout [48]. As a result, the produced dark matter relic abundance and baryon asymmetry can be diluted. Moreover one should take care that injected hadronic and electromagnetic energy does not disrupt the products of BBN.

In our model, since the freezeout of $N_{2}$ and $N_{3}$ occurs when they are nonrelativistic, the number density of these particles are highly suppressed. Therefore, the entropy and energy produced by the $N_{2,3}$ decays is negligible in terms of its cosmological impact. We illustrate this with an example; consider $M_{N}=1 \mathrm{GeV}$, and take the freezout temperature to be $T_{f} \sim M_{N} / 20 \sim 0.05 \mathrm{GeV}$, for which the number of degrees of freedom in the plasma is $g_{*} \cong 10$, and the decay rate is $\Gamma=0.01 \mathrm{~s}^{-1}$. The thermal number density of the HNLs at $T=T_{f}$ is $n_{N}=4\left(M_{N} T_{f} /(2 \pi)\right)^{3 / 2} \exp \left(-M_{N} / T_{f}\right)$, and its ratio to the entropy density at decoupling is denoted by $r_{N}=n_{N} / s$. Then ref. [48] shows that the entropy injected by HNL decays in this case is

$$
S \cong\left(1+3\left(\frac{2 \pi^{2} g_{*}}{45}\right)^{1 / 3} \frac{\left(r_{N} m_{N}\right)^{4 / 3}}{\left(M_{P} \Gamma\right)^{2 / 3}}\right)^{3 / 4} \cong 1+4 \times 10^{-9}
$$

where $S=1$ corresponds to the limit of no entropy production. This example shows that even when the lifetime is much longer than $1 \mathrm{~s}$, the abundance is too small to create any cosmological problem.

Previous studies show that even for decays as late as $100 \mathrm{~s}$, GeV-scale particles are only weakly constrained by BBN. Ref. [49] recently studied BBN constraints on models with late-decaying light particles, of mass up to $1 \mathrm{GeV}$. It shows that there are no constraints on electromagnetic injection for lifetimes less than $10^{4} \mathrm{~s}$, since nuclear photodissociation processes are suppressed at earlier times. Similarly, ref. [50] finds no significant bounds from hadronic injections for $\mathrm{GeV}$-scale particles decaying earlier than $100 \mathrm{~s}$.

\subsection{Lepton flavor violation bounds}

The mixing between light neutrinos and HNL's can lead to rare lepton-flavor violating processes, analogous to the well-studied case where $\mathrm{TeV}$-scale $\nu_{R}$ 's are responsible for the seesaw mass generation [51-56]. The decays $\mu \rightarrow e \gamma$ and $\mu \rightarrow 3 e$ are induced by the digrams shown in figure 5 . The most constraining process currently is $\mu \rightarrow e \gamma$, which has a branching ratio of [57]

$$
\operatorname{BR}(\mu \rightarrow e \gamma)=\frac{3 \alpha_{\mathrm{em}}}{32 \pi}\left|\sum_{i} U_{\mu i} U_{e i}^{*} \frac{M_{N}^{2}}{M_{W}^{2}}\right|^{2}=\frac{3 \alpha_{\mathrm{em}}}{32 \pi}\left|N N^{\dagger}\right|_{\mu e}^{2} \frac{M_{N}^{4}}{M_{W}^{4}}
$$


where $\alpha_{\mathrm{em}}$ is the electromagnetic fine structure constant and $\left|N N^{\dagger}\right|_{\mu e} \equiv\left|\sum_{i} N_{\mu i} N_{i e}^{\dagger}\right|$ with $N_{\alpha i}$ defined in eq. (4.5). For the case of normal neutrino mass hierarchy, our least restrictive bound based on EWPD, eq. (4.11), leads to $\left|\sum_{i} U_{\mu i} U_{e i}^{*}\right|<1 \times 10^{-3}$, and the prediction that $\operatorname{BR}(\mu \rightarrow e \gamma)<2.2 \times 10^{-15}$. This is still well below the current experimental bound of $4.2 \times 10^{-13}$ set by the MEG experiment [58].

From ref. [59] we find the branching ratio for $\mu \rightarrow 3 e$ in terms of $x \equiv M_{N}^{2} / M_{W}^{2} \ll 1$,

$$
\begin{aligned}
\mathrm{BR}(\mu \rightarrow 3 e) & \cong \frac{\alpha_{\mathrm{em}}^{2}}{16 \pi^{2} \sin ^{4} \theta_{W}}\left|\sum_{i} U_{\mu i} U_{e i}^{*}\right|^{2} x^{2}\left(0.6 \ln ^{2} x-0.2 \ln x+2.2\right) \\
& <1.6 \times 10^{-15}
\end{aligned}
$$

where the second line is the prediction using the value $\left|\sum_{i} U_{\mu i} U_{e i}^{*}\right|<1 \times 10^{-3}$ mentioned before. The experimental limit $\operatorname{BR}(\mu \rightarrow 3 e)<1 \times 10^{-12}$, set by the SINDRUM experiment [60], is weaker than that of the radiative decay.

Although the lepton-flavor-violating processes currently do not constrain the model better than EWPD constraints, experimental improvements could change this in the coming years. For example the Mu3e experiment may eventually probe $\mu \rightarrow 3 e$ down to the level of $10^{-16}$ branching ratio [61]. Moreover the process of $\mu N \rightarrow e N$ conversion in nuclei is expected to yield interesting limits in upcoming experiments, including Mu2e [62] at Fermilab and COMET [63] at KEK.

\section{Constraints on the singlet}

In recent years there have been intensive efforts to constrain the possible existence of light mediators connecting the SM to a hidden sector, the scalar singlet with Higgs portal being a prime example. The parameter space of $m_{s}$ and $\theta_{s}$ (the singlet-Higgs mixing angle) is constrained by a variety of beam-dump, collider and rare decay experiments, and by cosmology (big bang nucleosynthesis), astrophysics (supernova cooling) and dark matter direct searches. A large region of parameter space with $\theta_{s} \lesssim 10^{-3}$ and $m_{s} \lesssim 10 \mathrm{GeV}$ remains open, and parts of this will be targeted by the upcoming SHiP experiment [14]. In figure 6 we show some of the existing constraints, reproduced from ref. [69].

The KOTO experiment has searched for the rare decay $K_{L} \rightarrow \pi^{0} \nu \nu$ and set a new stringent upper limit of $3 \times 10^{-9}$ on its branching ratio [70,71]. Recently four candidate events above expected backgrounds were reported [18], far in excess of the standard model prediction ( $\left.\mathrm{BR}=3 \times 10^{-11}[34]\right)$. These could potentially be explained by the exotic decay mode $K_{L} \rightarrow \pi^{0} s$, if $s$ is sufficiently long-lived to escape detection, or if it decays invisibly. Such an interpretation has been previously considered in refs. [72-75]. In figure 6 , the $1 \sigma$ and $2 \sigma$ regions estimated in ref. [73] for explaining the KOTO excess are shown in blue. Parts of these regions are excluded by other experiments, notably NA62 [76] and E949 [65], but a range around $m_{s} \sim(120-160) \mathrm{MeV}$ and $\theta_{s} \sim(2-9) \times 10^{-4}$ remains viable.

The four plots in figure 6 pertain to different choices of the DM mass $m_{N^{\prime}}$, for the purpose of showing constraints from direct detection, that we describe in the following section. It can be seen that the region favored by the KOTO excess events is excluded by DM direct searches except for light DM, with $m_{N^{\prime}} \lesssim 2.5 \mathrm{GeV}$. 

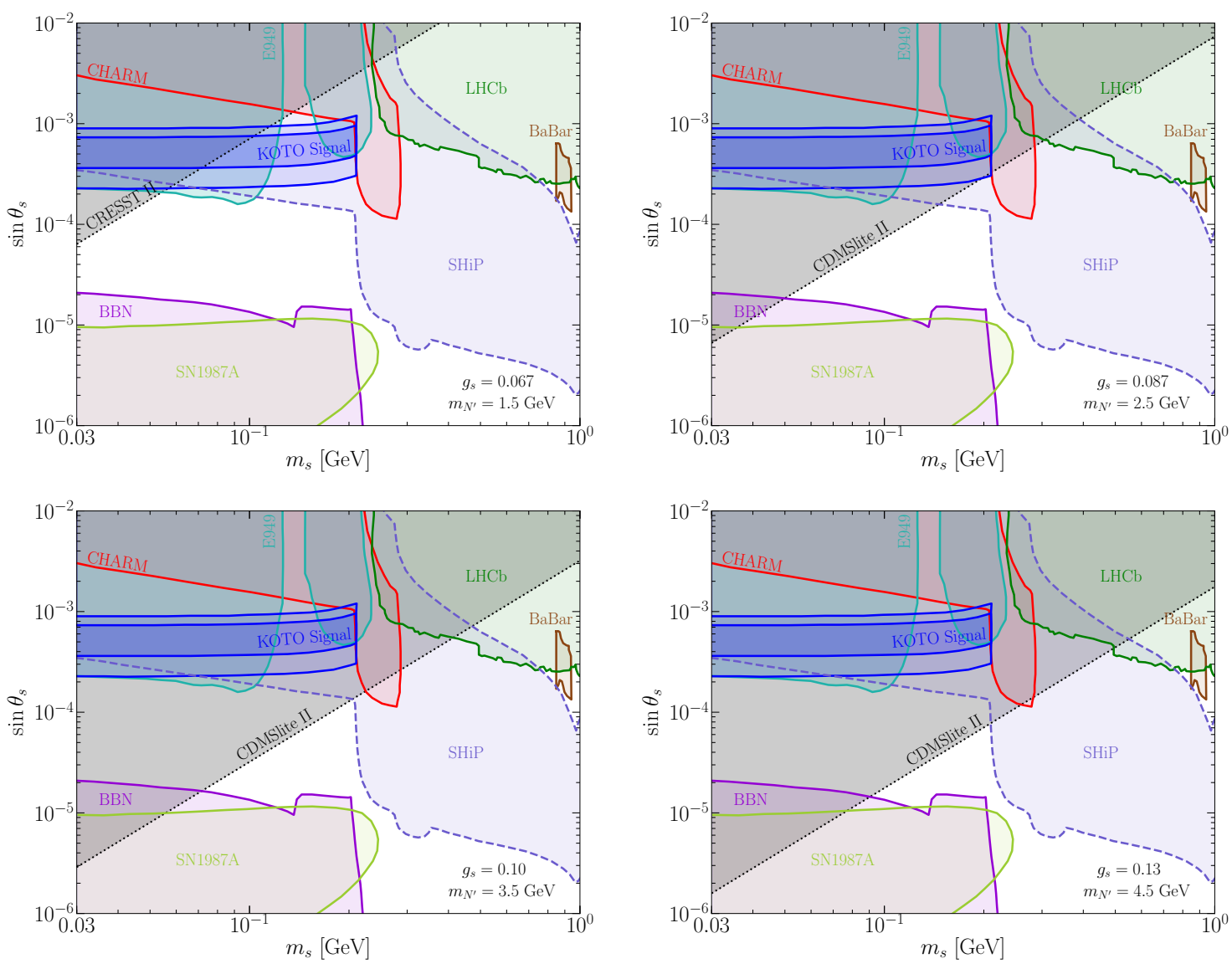

Figure 6. Constraints on a light singlet mediator, in the $m_{s}-\theta_{s}$ plane for the case $m_{s}<m_{N^{\prime}}$. The four plots consider different values of the DM mass $m_{N^{\prime}}=1.5,2.5,3.5,4.5 \mathrm{GeV}$, for which the direct detection constraints (black dotted line) differ; all other constraints are the same. The dark blue regions are favored at $1 \sigma$ and $2 \sigma$ for the KOTO anomaly. The red, cyan, green and brown regions are excluded by CHARM [64], E949 [65], LHCb [66] and BaBar experiments [67], respectively. The violet and light-green regions are excluded by BBN [68] and supernova data [69]. Sensitivity projection for the SHiP experiment is indicated by the dashed blue-gray boundary. The experimental bounds, along with the projected sensitivity, are taken from ref. [69].

\section{DM direct/indirect detection and self-interactions}

In general, the interactions of DM with nucleons versus with other particles are independent processes, whose cross sections need not be related. In our model however, both are mediated by exchange of the singlet $s$, so it is natural to consider them together.

\subsection{DM-nucleon scattering}

The mixing of $s$ with the Higgs boson leads to spin-independent DM interactions with nucleons. In particular, the cross section for scattering on nucleons is

$$
\sigma_{\mathrm{SI}}^{p}=\frac{g_{s}^{2} m_{N^{\prime}}^{2} m_{n}^{4} \sin ^{2} 2 \theta_{s} f_{n}^{2}}{4 \pi\left(m_{N^{\prime}}+m_{n}\right)^{2} \bar{v}^{2}}\left(\frac{1}{m_{h}^{2}}-\frac{1}{m_{s}^{2}}\right)^{2},
$$




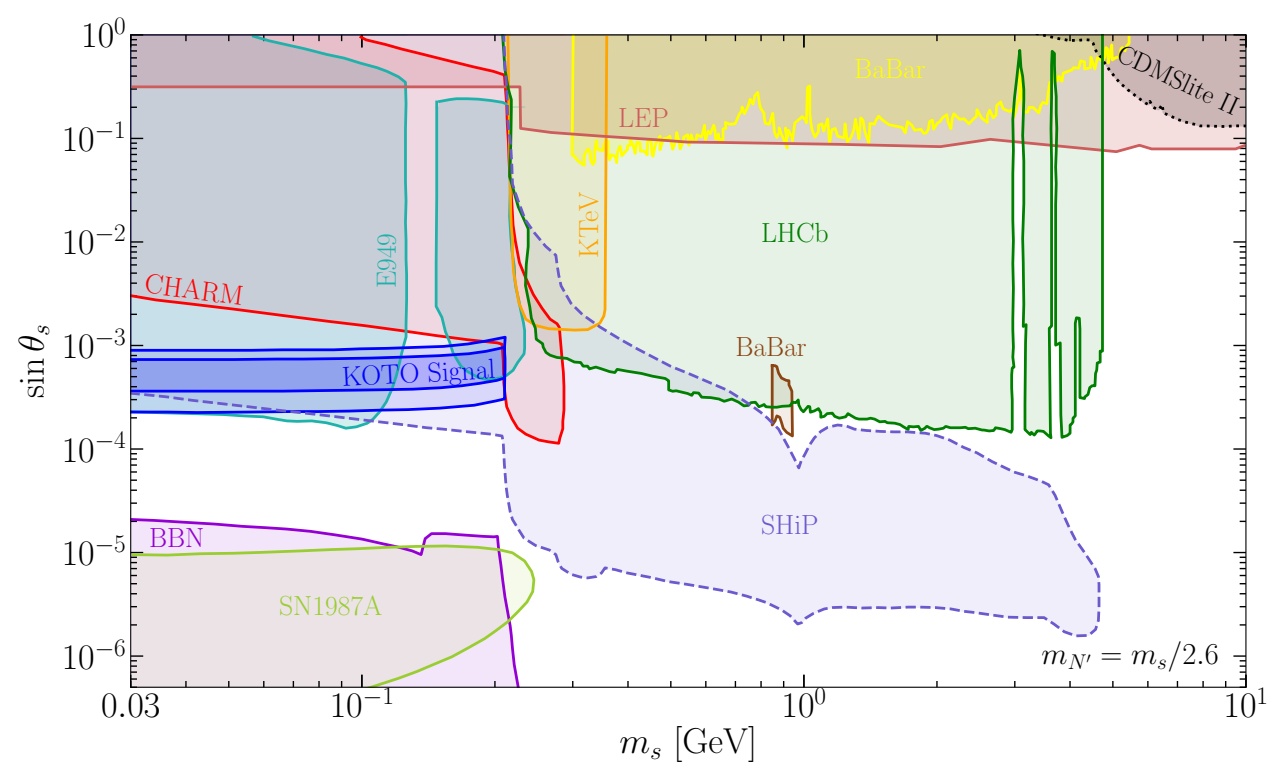

Figure 7. Constraints on a light singlet mediator, in the $m_{s}-\theta_{s}$ plane, for the case $m_{s}=2.6 m_{N^{\prime}}$. The experimental bounds, along with the projected sensitivity, are the same as in figure 6 and taken from ref. [69]. The strongest direct detection constraint derived for our model comes from CDMSlite II experiment [80] and is shown with the black dotted line.

where $m_{n}=0.938 \mathrm{GeV}$ is the nucleon mass, $f_{n}=0.30$ is the relative coupling of the Higgs to nucleons [77, 78], $m_{h}=125 \mathrm{GeV}$ is the SM-like Higgs mass, $m_{s}$ is the singlet mass and $\theta_{s}$ is the $s$ - $h$ mixing angle. (Recall that $\bar{v} \cong 174 \mathrm{GeV}$ is the complex Higgs field VEV.)

The strongest constraints from direct detection, in the mass range $m_{N^{\prime}}<4.5 \mathrm{GeV}$ predicted in our model, come from the experiments CRESST II [79], CDMSlite II [80] and LUX [81]. In the future these limits will be improved by SuperCDMS [82]. In all cases the sensitivity rapidly drops with lower DM mass because of the threshold for energy deposition. The DarkSide experiment [83] claims limits below those mentioned above, but their validity has been questioned in ref. [84], and we omit them from our analysis.

Recently ref. [85] cast doubts on the robustness of direct constraints on light dark matter in light of astrophysical uncertainties, especially that of the local escape velocity, that has been revisited using Gaia data [88]. It is claimed that the 2017 cross section bound from XENON1T [89] at DM mass $4 \mathrm{GeV}$ is uncertain by six orders of magnitude. We checked their results using DDCalc [86], finding only two orders of magnitude uncertainty. More importantly, the astrophysical uncertainty on the more relevant newer constraints is only a few percent (due to the much lower thresholds of those experiments), hence irrelevant.

For a given value of DM mass $m_{N^{\prime}}$, we can use the relic density constraint shown in figure 1 to determine the coupling $g_{s}$. Then the predicted direct detection cross section (6.1) leads to a constraint in the $m_{s}-\theta_{s}$ plane, that we plot as a dashed curve in figure 6 for the case $m_{s}<m_{N^{\prime}}$. As mentioned above, for larger values of $m_{N^{\prime}}$ the direct detection constraint is stronger, and the region favored by the KOTO anomaly is excluded.

For $m_{s}>m_{N^{\prime}}$, and particularly in the region where $m_{s} \gtrsim 2 m_{N^{\prime}}$, the direct detection constraints are shown in figure 7 for the case considered in the center panel of figure 1 , 
namely for $m_{s}=2.6 m_{N^{\prime}}$. These bounds are much weaker than those in figure 6 for any value of the DM mass $m_{N^{\prime}}$ because of the relatively larger assumed value of $m_{s}$, as can be seen from eq. (6.1).

\subsection{DM indirect detection}

Light dark matter models are typically constrained by indirect signals, like annihilation in the galaxy or the cosmic microwave background (CMB), enhanced by the relative large abundance of light DM. These signals are suppressed for asymmetric dark matter, by the absence of the symmetric component with which to annihilate, but DM accumulation in stars can provide significant constraints in the asymmetric case. Our model provides for a continuum of possibilities between the purely symmetric and asymmetric cases, depending on the strength of the coupling $g_{s}$ when $N^{\prime} \bar{N}^{\prime} \rightarrow s s$ is the dominant process, or a combination of $g_{s}$ and $m_{s}$ when $s$-channel annihilation dominates (recall figure 1).

However in our scenario there are several reasons for annihilation signals to be suppressed at late times, even in the symmetric regime. For the case where $N^{\prime} \bar{N}^{\prime} \rightarrow s s$ dominates, the cross section is $p$-wave, which significantly relaxes indirect constraints because of the low DM velocity at times much later than freezeout [87]. An exception can occur if the DM particles annihilate to form a bound state [95], which is $s$-wave, and leads to much stronger CMB constraints than the $p$-wave process. However this only occurs for relatively heavy $\mathrm{DM}$, with mass $\gtrsim 250 \mathrm{GeV}$.

In addition, the $p$-wave process we consider from $N^{\prime} \bar{N}^{\prime} \rightarrow f \bar{f}$ targets parameter space with $m_{s}>2 m_{N^{\prime}}$, such that $m_{s}$ is not too close to the threshold $2 m_{N^{\prime}}$. In this case the indirect signal is further suppressed, due to the low DM velocity in galaxies, $v \sim 10^{-3} c$, since the phase-space average of the annihilation cross section samples the resonant region much less than in the early universe during freezeout. Indeed, following refs. [87, 96], it is possible to estimate that for the values of $g_{s}$ and $m_{N^{\prime}}$ contained in figure 1 (center and right plots) the maximum ratio between the DM annihilation cross section today and that at the time of freezeout, given by eq. (3.16), is of order of $\sim 10^{-14}$, which leads today to $\langle\sigma v\rangle \lesssim 10^{-37} \mathrm{~cm}^{3} / \mathrm{s}$. Such a value is well below the most stringent indirect detection constraint for $p$-wave annihilating DM of mass $m_{N^{\prime}} \lesssim 4.5 \mathrm{GeV}$ [87].

Another possible signal that does not rely upon DM annihilation with itself, but rather on its interactions with standard model particles, is the effect of DM accumulation within stars. The most promising sites for capturing DM are neutron stars (NS's) because of their high density, which enhances the probability for DM particles to be captured and accumulate in the center of the NS during its lifetime.

For purely asymmetric DM, there is no DM annihilation in the NS core, and its accumulation may start to increase the star mass, destabilizing the delicate balance between the gravitational attraction and the Fermi pressure, and leading to the gravitational collapse of the NS into a black hole [90-92, 97]. However, this effect is only relevant in the case of bosonic DM, where there is no compensating increase in the Fermi pressure, leading to severe constraints on the DM-nucleon and DM-lepton scattering cross-sections based on the estimated age of the oldest NSs observed so far [92, 93]. These bounds do not apply to the present model because of the fermionic nature of our DM candidate, and its $\mathrm{GeV}$ 
mass scale. For fermionic asymmetric DM, the destabilization can occur only for DM with mass larger than the PeV scale and having attractive self-interactions [98].

In the case where DM is partially or purely symmetric, which occurs for smaller values of $m_{N^{\prime}}$ and $g_{s}$ in our model (recall figure 1), the accumulated DM inside the NS core can annihilate and the annihilation products might thermalize, heating up the star and contributing to its luminosity [94, 99]. The latter is also increased by DM kinetic heating from multiple DM scatterings with the NS constituents, namely neutrons, electrons and muons, and this effect is independent of whether the DM is symmetric or asymmetric [99, 100]. However, the expected NS surface temperature generated only by DM annihilation and scattering is too low to be detected by current infrared telescopes. A future detection by, for instance, the James Webb Space Telescope, would set the strongest bound on the DM-nucleon and DM-lepton scattering cross-sections for DM masses below the GeV scale, which would constrain our model [100].

Other limits on DM-nucleon interaction can in principle be derived from DM capture by white dwarfs (WD's) [97]. Similarly to NS's, asymmetric DM accumulating in the WD core might destabilize the latter and spark fusion reactions that precede a Type Ia supernova explosion [101]. However, in models where DM interactions with SM particles occur only via a light scalar mediator mixing with the Higgs boson, destabilization effects become important only for fermionic DM masses above $10^{6} \mathrm{GeV}$ [102].

On the other hand, DM scattering and annihilation can heat up WD's and contribute to their luminosity. The difference between the WD and the NS case is that very old WD's with low surface temperature have been observed, in particular within the M4 globular cluster $[103,104]$. Such observations have been used to claim very strong constraints on the DM-nucleon scattering cross section, $\sigma_{\mathrm{SI}} \lesssim 10^{-42}-10^{-43} \mathrm{~cm}^{2}$ for DM masses in the range $10^{-2}-10^{7} \mathrm{GeV}[105,106]$. These limits were derived based on the assumption that the DM density within the M4 globular cluster is as high as $10^{3} \mathrm{GeV} / \mathrm{cm}^{3}$, which can make the DM contribution to the WD luminosities as high as the observed values. However, as pointed out by ref. [105], the value of the DM density in globular clusters is highly uncertain and under debate. Although values of $10^{3} \mathrm{GeV} / \mathrm{cm}^{3}$ could be expected if globular clusters form within DM subhalos before falling into galactic halos [107], tidal stripping by subsequent mergers [108] provides a very efficient way of depleting DM in these systems, leaving them dominated today by just the stellar component [109]. The observation that the present-day dynamics of globular clusters can be explained without the need of DM suggests that these systems might form in molecular clouds in the gaseous disk of the galaxy instead of in DM overdensities [110-112]. It is therefore reasonable to assume that the DM density in the M4 globular cluster, which is about $2 \mathrm{kpc}$ away from us in the direction towards the galactic center, could be as low as in the solar neighborhood, $\sim 0.3 \mathrm{GeV} / \mathrm{cm}^{3}$. This lower value would reduce the saturated DM heating luminosity by approximately three orders of magnitude, well below the observed one, and lead to no bound on the DM-nucleon scattering cross section at all. More promising WD candidates might be found in globular clusters in dwarf spheroidal galaxies of the Milky Way, where a significant amount of DM may have survived tidal stripping [113]. 

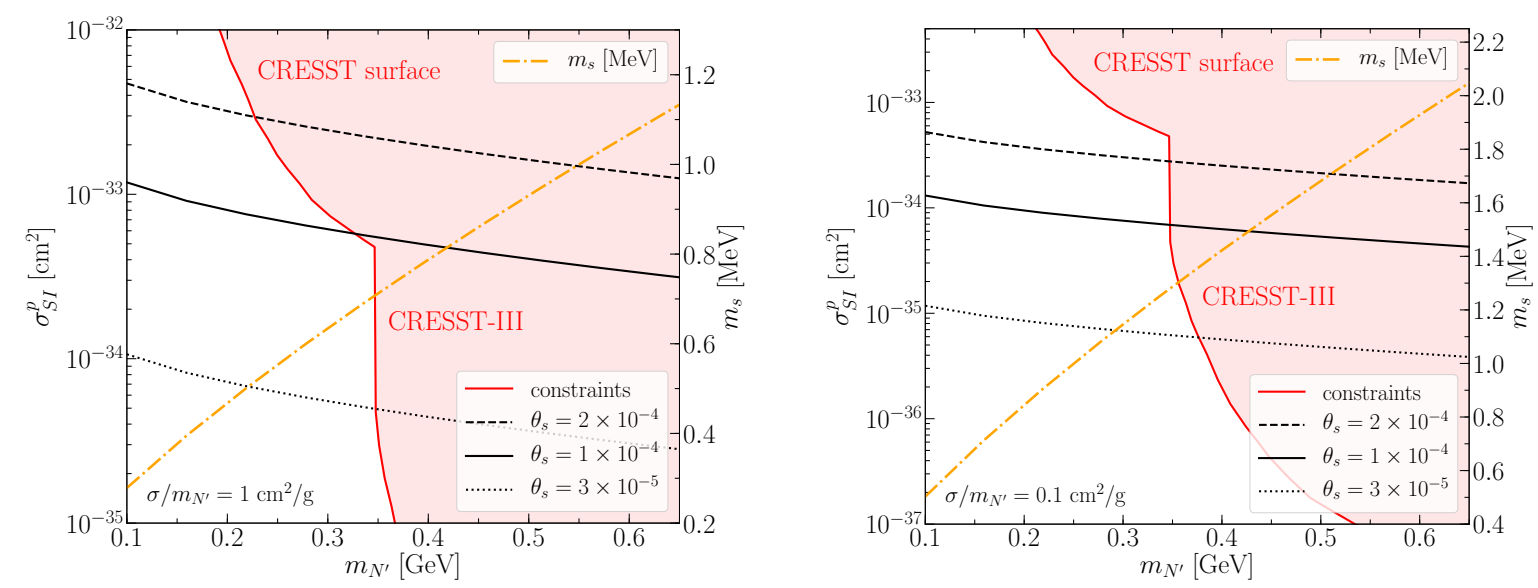

Figure 8. Predicted spin-independent cross section for DM scattering on nucleons versus DM mass $m_{N^{\prime}}$, assuming approximately symmetric DM with a self-interaction cross section of $\sigma / m_{N^{\prime}}$ $=1 \mathrm{~cm}^{2} / \mathrm{g}$ (left) or $0.1 \mathrm{~cm}^{2} / \mathrm{g}$ (right), for three choices of $\theta_{s}$ (dashed, solid black, dotted) and the envelope of experimental constraints (with the exception of DarkSide-50) copied from ref. [116] (solid red). Dash-dotted curve shows the singlet mass $m_{s}$ versus $m_{N^{\prime}}$.

\subsection{DM self-interactions}

Dark matter can also interact with itself by exchange of the $s$, which is of interest for addressing small-scale structure problems of collisionless cold dark matter (see ref. [114] for a review). Ref. [115] showed that the self-interaction cross section can be at an interesting level for solving these problems, while obtaining the right DM relic density, if both $m_{N^{\prime}}$ and $m_{s}$ are light,

$$
m_{s} \cong 1 \mathrm{MeV} \times \begin{cases}\left(\frac{m_{N^{\prime}}}{0.55 \mathrm{GeV}}\right)^{3 / 4}, & \sigma / m_{N^{\prime}}=1 \mathrm{~cm}^{2} / \mathrm{g} \\ \left(\frac{m_{N^{\prime}}}{0.25 \mathrm{GeV}}\right)^{3 / 4}, & \sigma / m_{N^{\prime}}=0.1 \mathrm{~cm}^{2} / \mathrm{g}\end{cases}
$$

These relations, valid for approximately symmetric DM, correspond to self-interaction cross section per mass in the range $\sigma / m_{N^{\prime}}=0.1-1 \mathrm{~cm}^{2} / \mathrm{g}$, that are relevant for suppressing cusps in density profiles of dwarf spheroidal to Milky Way-sized galaxies.

Such light singlets in the $\mathrm{MeV}$ mass range are strongly constrained by direct detection. The prediction (6.1) is modified by the fact that the momentum transfer $q$ is no longer negligible compared to $m_{s}$, hence $m_{s}^{2} \rightarrow m_{s}^{2}+q^{2}$ in eq. (6.1). We take $q=m_{N^{\prime}} v_{N^{\prime}}$ with DM velocity $v_{N^{\prime}} \sim 300 \mathrm{~km} / \mathrm{s}$ to account for this. Figure 6 shows that for low $m_{s}$ there is an allowed window for $\sin \theta_{s} \sim 2 \times\left(10^{-5}-10^{-4}\right)$ between the BBN and E949 constraints, which persists to smaller values of $m_{s} \gtrsim 1 \mathrm{MeV}$ before being excluded by BBN as $m_{s}$ falls below the threshold for $s \rightarrow e^{+} e^{-}$decay.

In figure 8 we show the predicted spin-independent cross section versus $m_{N^{\prime}}$ (black curves) for several choices of $\theta_{s}$ in the experimentally allowed range, fixing $g_{s}$ as in figure 1 to give the right relic density, and $m_{s}$ (orange dash-dotted curve) as a function of $m_{N^{\prime}}$ using (6.2). The plot on the left assumes the higher self-interaction cross section $\sigma / m=$ $1 \mathrm{~cm}^{2} / \mathrm{g}$. In this case, it is necessary to take the singlet mass $m_{s} \lesssim 0.7 \mathrm{MeV}$ and the DM 
mass $m_{N^{\prime}} \lesssim 0.3 \mathrm{GeV}$ to respect the direct detection limit. This is ruled out by BBN since the decay $s \rightarrow e^{+} e^{-}$are kinematically blocked, and will lead to overclosure by the singlets as $T$ falls below $m_{s}$. However, by adopting a lower self-interaction cross section $\sigma / m=0.1 \mathrm{~cm}^{2} / \mathrm{g}$ (right plot of figure 8), which may still be relevant for some of the smallscale structure issues, the allowed range of $m_{N^{\prime}}$ and $m_{s}$ is increased to somewhat larger values with $m_{s}>2 m_{e}$, which can be compatible with BBN.

The previous determination holds in the region $m_{N^{\prime}} \lesssim 3 \mathrm{GeV}$ where the $\mathrm{DM}$ is to a good approximation symmetric, corresponding to the linearly increasing branch of the relic density contour in figure 1 (left). For nearly asymmetric DM, the horizontal branch with $m_{N^{\prime}} \cong 4.5 \mathrm{GeV}$ applies. Instead of eq. (6.2), the desired self-interaction cross section requires a roughly linear relation $g_{s} \cong 0.75+4.43 m_{s} / \mathrm{GeV}$ (valid for $m_{s} \sim 0.2-0.3 \mathrm{GeV}$ ), that we determine by applying a Sommerfeld enhancement factor [117] to the tree-level, phase-space averaged transport scattering cross section given in ref. [115], and requiring that the resulting cross section is $\sigma / m_{N^{\prime}}=1 \mathrm{~b} / \mathrm{GeV} \cong 0.6 \mathrm{~cm}^{2} / \mathrm{g}$ for a mean DM velocity of $10 \mathrm{~km} / \mathrm{s}$, corresponding to dwarf spheroidal galaxies. To satisfy the CDMSLite II constraint $\sigma_{\mathrm{SI}}<1 \times 10^{-41} \mathrm{~cm}^{2}$ at $m_{N^{\prime}}=4.5 \mathrm{GeV}$ [118], it is necessary to take small mixing $\theta_{s} \lesssim 6 \times 10^{-6}$, since $g_{s} \sim 2$ for $m_{s} \sim 0.2-0.3 \mathrm{GeV}$, from imposing the desired value of $\sigma / m_{N^{\prime}}$

Hence we find two allowed regions for strong self-interactions, one marginal since $m_{s} \gtrsim 1 \mathrm{MeV}$ close to BBN limits, with $\sigma / m_{N^{\prime}} \sim 0.1 \mathrm{~cm}^{2} / \mathrm{g}$ at the low end of the range desired for small scale structure, and $m_{N^{\prime}} \sim 0.35 \mathrm{GeV}$. The other allows for a larger $\sigma / m_{N^{\prime}} \gtrsim 0.6 \mathrm{~cm}^{2} / \mathrm{g}$, with singlet parameters close to the SN1987A exclusion curve and $m_{N^{\prime}} \cong 4.5 \mathrm{GeV}$.

\section{$7 \quad$ Naturalness}

In our proposal, the flavor structure of neutrinos is controlled by the same matrix $\eta_{\nu, i j}$ that governs the HNL couplings, up to a proportionality constant, in the spirit of MFV. In order for DM to be stable, $\eta_{\nu, i j}$ must have rank two. The HNL mass matrix is proportional to the identity, up to corrections going as $\eta_{\nu}^{2}$. We do not provide any fundamental explanation of the origin of this structure; instead we content ourselves with the feature that it is technically natural in the sense of 't Hooft: all radiative corrections are consistent with our assumptions.

The stability of DM is most easily seen in the basis (4.14), (4.15), where $N^{\prime}$ obviously decouples from the SM leptons. We assume this coincides with the mass eigenbasis, which is consistent since there are no interactions that can induce mass-mixing between $N^{\prime}$ and the remaining $N_{i}$ 's. Self-energy corrections involving $s$ exchange are flavor-diagonal. Those involving Higgs and leptons in the loop leave $m_{N^{\prime}}$ unchanged, while renormalizing the $N_{i}$ mass matrix by

$$
M_{N} \delta_{i j} \rightarrow M_{N} \delta_{i j}+O(1) \times \eta_{\nu, i k} \frac{m_{\ell_{k}}}{16 \pi^{2}} \eta_{\nu, k j}^{\dagger}
$$

where $m_{\ell_{i}}$ are the charged lepton masses. Given the smallness of $\eta_{\nu} \lesssim 10^{-4}$, these corrections are unimportant. Similarly the one-loop corrections to $\eta_{\nu}$ are negligible,

$$
\eta_{\nu} \rightarrow \eta_{\nu}+\frac{O(1)}{16 \pi^{2}} \eta_{\nu} \eta_{\nu}^{\dagger} \eta_{\nu}
$$


and cannot induce couplings to $N^{\prime}$. The only particles to which $N^{\prime}$ couples are the singlet and the inflaton, eqs. (2.1), (3.9), and these interactions are assumed to be flavor-conserving at tree level. Flavor-changing corrections to $g_{s}$ and $g_{\phi}$ of $O\left(\eta_{\nu}^{2} /\left(16 \pi^{2}\right)\right) \times g_{s, \phi}$ arise at the one-loop level and are negligible for our purposes.

There remains the infamous naturalness problem of the Higgs mass (weak scale hierarchy). This problem was addressed in the context of the seesaw mechanism in ref. [119], where the weak scale was linked to that of the heavy Majorana neutrinos by radiative generation of the Higgs potential. A low scale for their masses is needed, $M_{\nu_{R}} \lesssim 10^{7} \mathrm{GeV}$ [120], which would require a low reheat temperature in our scenario, and consequently small coupling $g_{\phi} \lesssim 10^{-8}$. Although peculiarly small, this value would still be compatible with the requirements of technical naturalness since it can only be multiplicatively renormalized.

The very light singlet could pose an analogous problem of fine-tuning. The first threshold encountered when running the renormalization scale up from low values is that of $N_{i}$, which contributes of order $\delta m_{s} \sim g_{s} M_{N} /(4 \pi)$ to $m_{s}$. This can easily be compatible with the tree-level values of $m_{s}$ desired for large parts of the allowed parameter space (see figures 1 and 6).

Next one encounters the Higgs threshold, which further shifts $m_{s}$ through the coupling $\lambda_{h s}$. The correction is of order $\delta m_{s} \sim \sqrt{\lambda_{h s}} m_{h} / 4 \pi$ which is related to the mixing angle by $\theta_{s} \sim \lambda_{h s} v v_{s} / m_{h}^{2}$, where $v$ and $v_{s}$ are the respective VEVs of the Higgs and the singlet. In turn, $v_{s}$ depends upon the $s$ self-coupling through $m_{s}^{2} \sim \lambda_{s} v_{s}^{2}$. Using these and demanding that $\delta m_{s} \lesssim m_{s}$ gives the constraint $\sqrt{\lambda_{s}} \lesssim 16 \pi^{2} m_{s}^{3} v /\left(\theta_{s} m_{h}^{4}\right)$. This can always be satisfied by choosing small enough $\lambda_{s}$, but the latter has a minimum natural value given by its one-loop correction $\delta \lambda_{s} \sim g_{s}^{4} /\left(16 \pi^{2}\right) .{ }^{7}$ Putting all of these together, we get a naturalness bound on the singlet mixing angle

$$
\theta_{s} \lesssim\left(\frac{4 \pi m_{s}}{m_{h}}\right)^{3}\left(\frac{1}{\sqrt{\lambda_{h}} g_{s}^{2}}\right) \sim 0.008
$$

(taking $m_{s} \sim 0.3 \mathrm{GeV}$ and $g_{s} \sim 0.1$ ) which is compatible with the regions of interest for future discovery, including the anomalous KOTO events. Thus, somewhat surprisingly, the light scalar does not introduce a new hierarchy problem analogous to that of the Higgs mass, due to its relatively weak couplings.

We do not address the smallness of $\theta_{Q C D}$ in our "theory of everything," which was a motivation for refs. [7,9] to choose the QCD axion as their dark matter candidate. This neglect is consistent with our philosophy of focusing on technical naturalness rather than aesthetic values of couplings, since $\theta_{Q C D}$ is known to be highly stable against radiative corrections [121].

\section{Conclusions}

It is interesting to construct scenarios that link the different particle physics ingredients known to be missing from the standard model, since it can lead to distinctive predictions.

\footnotetext{
${ }^{7}$ There is also a one-loop correction of order $\lambda_{h s}^{2} / 16 \pi^{2}$, but this leads to a weaker bound on $\theta_{s}$ than (7.3).
} 
Here we have constructed a minimal scenario that explains inflation, baryogenesis, dark matter and neutrino masses, is highly predictive, and can be tested in numerous experimental searches for heavy neutral leptons, light dark matter, and light scalar mediators. At low energies, the only new particles are three quasi-Dirac HNLs, one of which is DM (and exactly Dirac), and a light singlet scalar.

One prediction of the model is that no new source of CP-violation is required for baryogenesis, which occurs through a novel form of leptogenesis here. In contrast to ordinary leptogenesis, the asymmetry is formed during inflation, and the right-handed neutrinos that generate light neutrino masses are too heavy to be produced during reheating. $\mathrm{CP}$ is spontaneously broken by the inflaton VEV during inflation, and the light HNLs transmit the lepton asymmetry from the inflaton to the SM. In ref. [12] it was shown that observable isocurvature perturbations can arise, depending on the inflaton potential and initial conditions. In the present model, these would appear as correlated dark matter isocurvature and adiabatic perturbations.

Another prediction is that the two unstable HNLs $N_{i}$ should be degenerate to very high precision with the dark matter $N^{\prime}$, split only by the correction (7.1) of order $10^{-2} \mathrm{eV}$. Similarly, the $N_{i}$ are Dirac particles to a very good approximation, with a lepton-violating Majorana mass of order $10^{-6} \mathrm{eV}$. This is too small to be detectable in neutrinoless double beta decay, but large enough to allow for a distinctive signature of lepton violation through $N-\bar{N}$ oscillations. The two $N_{i}$ HNLs can mix strongly enough with SM neutrinos to be discoverable at upcoming experiments like SHiP. The stability of $N^{\prime}$ is directly linked to the masslessness of the lightest neutrino. This connection could be relaxed by slightly modifying the assumption that the HNL couplings are aligned with light neutrino masses through eq. (2.4), without spoiling other features of our model. We further showed that lepton-flavor-violating decays like $\mu \rightarrow e \gamma$ and $\mu \rightarrow 3 e$ may be generated by HNL exchange in loops, at a level that can be detected in future experiments.

In our framework, the dark matter $N^{\prime}$ is partially asymmetric, and has a mass bounded by $m_{N^{\prime}} \lesssim 4.5 \mathrm{GeV}$. The bound is saturated when $N^{\prime}$ is purely asymmetric, and its mass is determined by the observed value of the baryon asymmetry. Light DM can be accommodated by taking small values of the coupling $g_{s}$ between $N^{\prime}$ and the singlet $s$, which controls $N^{\prime} \bar{N}^{\prime} \rightarrow$ ss annihilation; see figure 1 . In the mass range $(1-4.5) \mathrm{GeV}$, significant constraints are already placed by direct DM searches.

The light scalar singlet, whose mass must be less than $m_{N^{\prime}}$ for efficient $N^{\prime} \bar{N}^{\prime} \rightarrow s s$ annihilation, can lead to striking signatures. For example the decay $K_{L} \rightarrow \pi s$ can explain anomalous excess events recently observed by the KOTO experiment, but only if $m_{N^{\prime}} \lesssim$ $2.5 \mathrm{GeV}$; otherwise direct detection constraints rule out this mode at the level suggested by the KOTO events, where $m_{s} \sim(100-200) \mathrm{MeV}$ and $s$ mixes with the Higgs at the level $\theta_{s} \sim 5 \times 10^{-4}$. (The preferred parameter region for the KOTO anomaly is only a small part of the full allowed space of our model.) In a different part of parameter space with $m_{s} \sim(0.2-0.3) \mathrm{GeV}, m_{N^{\prime}} \cong 4.5 \mathrm{GeV}$ and $\theta_{s} \lesssim 6 \times 10^{-6}$, the singlet mediates DM self-interactions with a cosmologically interesting cross section, $\sigma / m_{N^{\prime}} \sim 0.6 \mathrm{~cm}^{2} / \mathrm{g}$. 


\section{Acknowledgments}

We thank J. Kopp for an insightful question that inspired this work, and G. AlonsoÁlvarez, J. Bramante, T. Bringmann, L. Di Ciaccio, M. Fairbairn, E. Fernandez-Martinez, F. Kahlhoefer, C. Lacasta, E. Migliore, D. Morrissey, S. Petcov, M. Reina-Campos, P. Scott, J.-L. Tastet and J. Timmermans for helpful correspondence. Our research is supported by NSERC (Natural Sciences and Engineering Research Council, Canada). MP is supported by the Arthur B. McDonald Institute for Canadian astroparticle physics research.

\section{A Decay rate for $N_{i} \rightarrow \nu \ell^{+} \ell^{-}$}

The matrix element for the process $N_{i} \rightarrow \nu_{\beta} \ell_{\beta}^{+} \ell_{\alpha}^{-}$, where $\alpha, \beta=e, \mu, \tau$, is

$$
\mathcal{M}=\frac{g_{w}^{2}}{8 M_{W}^{2}}\left[\bar{u}\left(p_{\ell_{\alpha}^{-}}\right) \gamma^{\mu}\left(1-\gamma^{5}\right) u\left(p_{N_{i}}\right) U_{i \alpha}^{*}\right]\left[\bar{u}\left(p_{\nu_{\beta}}\right) \gamma_{\mu}\left(1-\gamma^{5}\right) u\left(p_{\ell_{\beta}^{+}}\right)\right]
$$

whose square reduces to

$$
\left\langle|\mathcal{M}|^{2}\right\rangle=\frac{G_{F}^{2}}{16}\left|U_{i \alpha}\right|^{2} M_{i} E_{\beta}\left[\frac{M_{i}^{2}+m_{\beta}^{2}-m_{\alpha}^{2}}{2}-M_{i} E_{\beta}\right]
$$

after averaging over the initial spin, summing over final spins and setting $m_{\nu_{\beta}}=0$. Here, $G_{F}$ is the Fermi constant, $E_{\beta}$ is the energy of $\ell_{\beta}^{+}$and we have defined for simplicity $M_{i} \equiv M_{N_{i}}, m_{\alpha} \equiv m_{\ell_{\alpha}^{-}}$and $m_{\beta} \equiv m_{\ell_{\beta}^{+}}$. The decay rate $\Gamma$ can be obtained by plugging eq. (A.2) in the standard decay formula (see ref. [34]) and computing the three-body phase space integral. The common assumption made in the literature is to consider $m_{\beta}=0$, which is well motivated for $\alpha=e, \mu$ and $\beta=\mu, e$. In these cases, the decay rate is $[44,46]$

$$
\Gamma=\frac{G_{F}^{2} M_{i}^{5}}{192 \pi^{3}}\left|U_{i \alpha}\right|^{2}\left(1-8 x_{\alpha}^{2}+8 x_{\alpha}^{6}-x_{\alpha}^{8}-12 x_{\alpha}^{4} \log \left(x_{\alpha}^{2}\right)\right)
$$

where $x_{\alpha}=m_{\alpha} / M_{i}$. Such a simplified formula does not hold for $\alpha=\mu, \tau$ and $\beta=\tau, \mu$, where the muon mass is not negligible compared to the tau mass. The general expression reads

$$
\begin{aligned}
\Gamma= & \frac{G_{F}^{2} M_{i}^{5}}{192 \pi^{3}}\left|U_{i \alpha}\right|^{2}\left\{12\left|x_{\beta}^{2}-x_{\alpha}^{2}\right|\left(x_{\beta}^{2}+x_{\alpha}^{2}\right)\right. \\
& \times \log \left[\frac{x_{\beta}^{2}+x_{\alpha}^{2}-\left(x_{\beta}^{2}-x_{\alpha}^{2}\right)^{2}-\left|x_{\beta}^{2}-x_{\alpha}^{2}\right| \sqrt{\left(1-\left(x_{\beta}-x_{\alpha}\right)^{2}\right)\left(1-\left(x_{\beta}+x_{\alpha}\right)^{2}\right)}}{2 x_{\beta} x_{\alpha}}\right] \\
& -12\left[x_{\beta}^{4}+x_{\alpha}^{4}-2 x_{\beta}^{4} x_{\alpha}^{4}\right] \log \left[\frac{1-x_{\beta}^{2}-x_{\alpha}^{2}-\sqrt{\left(1-\left(x_{\beta}-x_{\alpha}\right)^{2}\right)\left(1-\left(x_{\beta}+x_{\alpha}\right)^{2}\right)}}{2 x_{\beta} x_{\alpha}}\right] \\
& +\sqrt{\left(1-\left(x_{\beta}-x_{\alpha}\right)^{2}\right)\left(1-\left(x_{\beta}+x_{\alpha}\right)^{2}\right)} \\
& \left.\times\left[1-7\left(x_{\beta}^{2}+x_{\alpha}^{2}\right)\left(1+x_{\beta}^{2} x_{\alpha}^{2}\right)-7\left(x_{\beta}^{4}+x_{\alpha}^{4}\right)+12 x_{\beta}^{2} x_{\alpha}^{2}+x_{\beta}^{6}+x_{\alpha}^{6}\right]\right\}
\end{aligned}
$$

where $x_{\alpha} \equiv m_{\alpha} / M_{i}$ and $x_{\beta} \equiv m_{\beta} / M_{i}$. It is easy to check that this formula reduces to eq. (A.3) in the limit $m_{\beta} \rightarrow 0$. 
Open Access. This article is distributed under the terms of the Creative Commons Attribution License (CC-BY 4.0), which permits any use, distribution and reproduction in any medium, provided the original author(s) and source are credited.

\section{References}

[1] G. Alonso-Álvarez, G. Elor, A.E. Nelson and H. Xiao, A supersymmetric theory of baryogenesis and sterile sneutrino dark matter from B mesons, JHEP 03 (2020) 046 [arXiv: 1907.10612] [INSPIRE].

[2] T. Asaka and M. Shaposhnikov, The $\nu M S M$, dark matter and baryon asymmetry of the universe, Phys. Lett. B 620 (2005) 17 [hep-ph/0505013] [INSPIRE].

[3] M. Shaposhnikov and I. Tkachev, The $\nu M S M$, inflation and dark matter, Phys. Lett. B 639 (2006) 414 [hep-ph/0604236] [INSPIRE].

[4] F.L. Bezrukov and M. Shaposhnikov, The standard model Higgs boson as the inflaton, Phys. Lett. B 659 (2008) 703 [arXiv:0710.3755] [INSPIRE].

[5] S. Choubey and A. Kumar, Inflation and dark matter in the inert doublet model, JHEP 11 (2017) 080 [arXiv: 1707.06587] [INSPIRE].

[6] D. Borah, P.S.B. Dev and A. Kumar, TeV scale leptogenesis, inflaton dark matter and neutrino mass in a scotogenic model, Phys. Rev. D 99 (2019) 055012 [arXiv:1810.03645] [INSPIRE].

[7] G. Ballesteros, J. Redondo, A. Ringwald and C. Tamarit, Unifying inflation with the axion, dark matter, baryogenesis and the seesaw mechanism, Phys. Rev. Lett. 118 (2017) 071802 [arXiv: 1608.05414] [INSPIRE].

[8] M.A. Shifman, A.I. Vainshtein and V.I. Zakharov, Can confinement ensure natural CP invariance of strong interactions?, Nucl. Phys. B 166 (1980) 493 [InSPIRE].

[9] A. Salvio, A simple motivated completion of the standard model below the Planck scale: axions and right-handed neutrinos, Phys. Lett. B 743 (2015) 428 [arXiv:1501.03781] [INSPIRE].

[10] A. Salvio, Critical Higgs inflation in a viable motivated model, Phys. Rev. D 99 (2019) 015037 [arXiv: 1810.00792] [INSPIRE].

[11] I. Affleck and M. Dine, A new mechanism for baryogenesis, Nucl. Phys. B 249 (1985) 361 [INSPIRE].

[12] J.M. Cline, M. Puel and T. Toma, Affleck-Dine inflation, Phys. Rev. D 101 (2020) 043014 [arXiv: 1909.12300] [INSPIRE].

[13] Planck collaboration, Planck 2018 results. X. Constraints on inflation, arXiv: 1807.06211 [INSPIRE].

[14] S. Alekhin et al., A facility to search for hidden particles at the CERN SPS: the SHiP physics case, Rept. Prog. Phys. 79 (2016) 124201 [arXiv: 1504.04855] [INSPIRE].

[15] D. Curtin et al., Long-lived particles at the energy frontier: the MATHUSLA physics case, Rept. Prog. Phys. 82 (2019) 116201 [arXiv: 1806.07396] [InSPIRE].

[16] J.L. Feng, I. Galon, F. Kling and S. Trojanowski, ForwArd Search ExpeRiment at the LHC, Phys. Rev. D 97 (2018) 035001 [arXiv:1708.09389] [InSPIRE]. 
[17] V.V. Gligorov, S. Knapen, M. Papucci and D.J. Robinson, Searching for long-lived particles: a compact detector for exotics at LHCb, Phys. Rev. D 97 (2018) 015023 [arXiv: 1708.09395] [INSPIRE].

[18] S. Shinohara, Search for the rare decay $K_{L} \rightarrow \pi^{0} \nu \bar{\nu} J-P A R C$ KOTO experiment, talk given at the International Conference on Kaon Physics (KAON2019), September 10-13, Perugia, Italy (2009).

[19] G. D'Ambrosio, G.F. Giudice, G. Isidori and A. Strumia, Minimal flavor violation: an effective field theory approach, Nucl. Phys. B 645 (2002) 155 [hep-ph/0207036] [InSPIRE].

[20] K.D. Lozanov and M.A. Amin, End of inflation, oscillons and matter-antimatter asymmetry, Phys. Rev. D 90 (2014) 083528 [arXiv: 1408.1811] [INSPIRE].

[21] B.A. Campbell, S. Davidson, J.R. Ellis and K.A. Olive, On the baryon, lepton flavor and right-handed electron asymmetries of the universe, Phys. Lett. B 297 (1992) 118 [hep-ph/9302221] [INSPIRE].

[22] J.M. Cline, Constraints on almost Dirac neutrinos from neutrino-anti-neutrino oscillations, Phys. Rev. Lett. 68 (1992) 3137 [INSPIRE].

[23] T. Bringmann, J.M. Cline and J.M. Cornell, Baryogenesis from neutron-dark matter oscillations, Phys. Rev. D 99 (2019) 035024 [arXiv: 1810.08215] [InSPIRE].

[24] S. Tulin, H.-B. Yu and K.M. Zurek, Oscillating asymmetric dark matter, JCAP 05 (2012) 013 [arXiv: 1202.0283] [INSPIRE].

[25] M. Cirelli, P. Panci, G. Servant and G. Zaharijas, Consequences of DM/antiDM oscillations for asymmetric WIMP dark matter, JCAP 03 (2012) 015 [arXiv:1110.3809] [INSPIRE].

[26] J.A. Harvey and M.S. Turner, Cosmological baryon and lepton number in the presence of electroweak fermion number violation, Phys. Rev. D 42 (1990) 3344 [INSPIRE].

[27] Planck collaboration, Planck 2018 results. VI. Cosmological parameters, arXiv: 1807.06209 [INSPIRE].

[28] P. Gondolo and G. Gelmini, Cosmic abundances of stable particles: improved analysis, Nucl. Phys. B 360 (1991) 145 [INSPIRE].

[29] M.L. Graesser, I.M. Shoemaker and L. Vecchi, Asymmetric WIMP dark matter, JHEP 10 (2011) 110 [arXiv: 1103.2771] [INSPIRE].

[30] R. Iengo, Sommerfeld enhancement: general results from field theory diagrams, JHEP 05 (2009) 024 [arXiv: 0902.0688] [INSPIRE].

[31] M. Drewes and B. Garbrecht, Combining experimental and cosmological constraints on heavy neutrinos, Nucl. Phys. B 921 (2017) 250 [arXiv:1502.00477] [INSPIRE].

[32] S. Antusch and O. Fischer, Non-unitarity of the leptonic mixing matrix: Present bounds and future sensitivities, JHEP 10 (2014) 094 [arXiv: 1407.6607] [INSPIRE].

[33] E. Fernandez-Martinez, J. Hernandez-Garcia and J. Lopez-Pavon, Global constraints on heavy neutrino mixing, JHEP 08 (2016) 033 [arXiv: 1605.08774] [INSPIRE].

[34] Particle Data Group collaboration, Review of particle physics, Phys. Rev. D 98 (2018) 030001.

[35] D.A. Bryman and R. Shrock, Constraints on sterile neutrinos in the MeV to GeV mass range, Phys. Rev. D 100 (2019) 073011 [arXiv:1909.11198] [InSPIRE]. 
[36] E. Goudzovski, HNL production and exotic searches at NA62, talk given at the International Conference on Kaon Physics (KAON2019), September 10-13, Perugia, Italy (2009).

[37] FCC-ee study Team collaboration, Search for heavy right handed neutrinos at the FCC-ee, Nucl. Part. Phys. Proc. 273-275 (2016) 1883 [arXiv:1411.5230] [inSPIRE].

[38] I. Krasnov, DUNE prospects in the search for sterile neutrinos, Phys. Rev. D 100 (2019) 075023 [arXiv: 1902.06099] [inSPIRE].

[39] SHIP collaboration, Sensitivity of the SHiP experiment to heavy neutral leptons, JHEP 04 (2019) 077 [arXiv: 1811.00930] [INSPIRE].

[40] DELPHI collaboration, Search for neutral heavy leptons produced in $Z$ decays, Z. Phys. C 74 (1997) 57 [Erratum ibid. C 75 (1997) 580] [INSPIRE].

[41] CHARM collaboration, A search for decays of heavy neutrinos in the mass range $0.5 \mathrm{GeV}$ to 2.8 GeV, Phys. Lett. B 166 (1996) 473.

[42] NuTeV, E815 collaboration, Search for neutral heavy leptons in a high-energy neutrino beam, Phys. Rev. Lett. 83 (1999) 4943 [hep-ex/9908011] [InSPIRE].

[43] J.-L. Tastet and I. Timiryasov, Dirac vs. Majorana HNLs (and their oscillations) at SHiP, JHEP 04 (2020) 005 [arXiv: 1912.05520] [INSPIRE].

[44] L.M. Johnson, D.W. McKay and T. Bolton, Extending sensitivity for low mass neutral heavy lepton searches, Phys. Rev. D 56 (1997) 2970 [hep-ph/9703333] [INSPIRE].

[45] P.B. Pal and L. Wolfenstein, Radiative decays of massive neutrinos, Phys. Rev. D 25 (1982) 766 [INSPIRE].

[46] D. Gorbunov and M. Shaposhnikov, How to find neutral leptons of the $\nu M S M$ ?, JHEP 10 (2007) 015 [Erratum ibid. 11 (2013) 101] [arXiv: 0705.1729] [INSPIRE].

[47] E949 collaboration, Search for heavy neutrinos in $K^{+} \rightarrow \mu^{+} \nu_{H}$ decays, Phys. Rev. D 91 (2015) 052001 [Erratum ibid. D 91 (2015) 059903] [arXiv:1411.3963] [INSPIRE].

[48] F. Bezrukov, H. Hettmansperger and M. Lindner, keV sterile neutrino Dark Matter in gauge extensions of the Standard Model, Phys. Rev. D 81 (2010) 085032 [arXiv:0912.4415] [INSPIRE].

[49] J. Coffey, L. Forestell, D.E. Morrissey and G. White, Cosmological bounds on sub-GeV dark vector bosons from electromagnetic energy injection, arXiv:2003.02273 [INSPIRE].

[50] M. Pospelov and J. Pradler, Metastable GeV-scale particles as a solution to the cosmological lithium problem, Phys. Rev. D 82 (2010) 103514 [arXiv: 1006.4172] [INSPIRE].

[51] S.T. Petcov, The processes $\mu \rightarrow e \gamma, \mu \rightarrow e e \bar{e}, \nu^{\prime} \rightarrow \nu \gamma$ in the Weinberg-Salam model with neutrino mixing, Sov. J. Nucl. Phys. 25 (1977) 340 [Erratum ibid. 25 (1977) 698] [INSPIRE].

[52] S.M. Bilenky, S.T. Petcov and B. Pontecorvo, Lepton mixing, $\mu \rightarrow e+\gamma$ decay and neutrino oscillations, Phys. Lett. 67B (1977) 309 [INSPIRE].

[53] T.P. Cheng and L.-F. Li Muon number nonconservation in gauge theories, in the proceedings of the Orbis Scientiae 1977: Deeper Pathways in High-Energy Physics, 17-21 January, Coral Gables, U.S.A. (1977).

[54] W.J. Marciano and A.I. Sanda, Exotic decays of the muon and heavy leptons in gauge theories, Phys. Lett. 67B (1977) 303 [InSPIRE]. 
[55] B.W. Lee, S. Pakvasa, R.E. Shrock and H. Sugawara, Muon and electron number nonconservation in a V-A gauge model, Phys. Rev. Lett. 38 (1977) 937 [Erratum ibid. 38 (1977) 1230] [INSPIRE].

[56] B.W. Lee and R.E. Shrock, Natural suppression of symmetry violation in gauge theories: muon- epton and electron lepton number nonconservation, Phys. Rev. D 16 (1977) 1444 [INSPIRE].

[57] L. Calibbi and G. Signorelli, Charged lepton flavour violation: an experimental and theoretical introduction, Riv. Nuovo Cim. 41 (2018) 71 [arXiv:1709.00294] [INSPIRE].

[58] MEG collaboration, Search for the lepton flavour violating decay $\mu^{+} \rightarrow \mathrm{e}^{+} \gamma$ with the full dataset of the MEG experiment, Eur. Phys. J. C 76 (2016) 434 [arXiv:1605.05081] [INSPIRE].

[59] D.N. Dinh, A. Ibarra, E. Molinaro and S.T. Petcov, The $\mu-e$ conversion in nuclei, $\mu \rightarrow e \gamma, \mu \rightarrow 3 e$ decays and TeV scale see-saw scenarios of neutrino mass generation, JHEP 08 (2012) 125 [Erratum ibid. 09 (2013) 023] [arXiv:1205.4671] [INSPIRE].

[60] SINDRUM collaboration, Search for the decay $\mu^{+} \rightarrow e^{+} e^{+} e^{-}$, Nucl. Phys. B 299 (1988) 1.

[61] Mu3E collaboration, Searching for lepton flavour violation with the Mu3e experiment, PoS (NuFact2017) 105 [arXiv: 1802.09851] [INSPIRE].

[62] Mu2E collaboration, Charged lepton flavour violation using intense muon beams at future facilities, FERMILAB-FN-1064 (2019).

[63] COMET collaboration, COMET status and plans, EPJ Web Conf. 212 (2019) 01006 [INSPIRE].

[64] CHARM collaboration, Search for axion like particle production in $400 \mathrm{GeV}$ proton-copper interactions, Phys. Lett. B 157 (1985) 458.

[65] BNL-E949 collaboration, Study of the decay $K^{+} \rightarrow \pi^{+} \nu \bar{\nu}$ in the momentum region $140<P_{\pi}<199 \mathrm{MeV} / \mathrm{c}$, Phys. Rev. D 79 (2009) 092004 [arXiv:0903.0030] [InSPIRE].

[66] LHCb collaboration, Differential branching fraction and angular analysis of the $B^{+} \rightarrow K^{+} \mu^{+} \mu^{-}$decay, JHEP 02 (2013) 105 [arXiv: 1209.4284] [INSPIRE].

[67] BABAR collaboration, Search for long-lived particles in $e^{+} e^{-}$collisions, Phys. Rev. Lett. 114 (2015) 171801 [arXiv: 1502.02580] [INSPIRE].

[68] A. Fradette and M. Pospelov, BBN for the LHC: constraints on lifetimes of the Higgs portal scalars, Phys. Rev. D 96 (2017) 075033 [arXiv:1706.01920] [InSPIRE].

[69] M.W. Winkler, Decay and detection of a light scalar boson mixing with the Higgs boson, Phys. Rev. D 99 (2019) 015018 [arXiv: 1809.01876] [INSPIRE].

[70] KOTO collaboration, Status on the search for $K_{L}^{0} \rightarrow \pi^{0} \nu \bar{\nu}$ with the KOTO experiment, arXiv: 1910.07585 [INSPIRE].

[71] KOTO collaboration, New results on the search for rare kaon events with the KOTO detector, arXiv:1910.07148 [INSPIRE].

[72] T. Kitahara et al., New physics implications of recent search for $K_{L} \rightarrow \pi^{0} \nu \bar{\nu}$ at KOTO, Phys. Rev. Lett. 124 (2020) 071801 [arXiv: 1909.11111] [INSPIRE].

[73] D. Egana-Ugrinovic, S. Homiller and P. Meade, Light scalars and the KOTO anomaly, arXiv: 1911.10203 [INSPIRE]. 
[74] P.S.B. Dev, R.N. Mohapatra and Y. Zhang, Constraints on long-lived light scalars with flavor-changing couplings and the KOTO anomaly, Phys. Rev. D 101 (2020) 075014 [arXiv:1911.12334] [INSPIRE].

[75] J. Liu, N. McGinnis, C.E.M. Wagner and X.-P. Wang, A light scalar explanation of $(g-2)_{\mu}$ and the KOTO anomaly, JHEP 04 (2020) 197 [arXiv:2001.06522] [INSPIRE].

[76] G. Ruggiero, Latest measurement of $K^{+} \rightarrow \pi^{+} \nu \bar{\nu}$ with the NA62 experiment at CERN, talk given at the International Conference on Kaon Physics (KAON2019), September 10-13, Perugia, Italy (2009).

[77] J.M. Cline, K. Kainulainen, P. Scott and C. Weniger, Update on scalar singlet dark matter, Phys. Rev. D 88 (2013) 055025 [Erratum ibid. D 92 (2015) 039906] [arXiv:1306.4710] [INSPIRE].

[78] J. Ellis, N. Nagata and K.A. Olive, Uncertainties in WIMP dark matter scattering revisited, Eur. Phys. J. C 78 (2018) 569 [arXiv:1805.09795] [INSPIRE].

[79] CRESST collaboration, Results on light dark matter particles with a low-threshold CRESST-II detector, Eur. Phys. J. C 76 (2016) 25 [arXiv:1509.01515] [InSPIRE].

[80] SupERCDMS collaboration, New results from the search for low-mass weakly interacting massive particles with the CDMS low ionization threshold experiment, Phys. Rev. Lett. 116 (2016) 071301 [arXiv:1509.02448] [INSPIRE].

[81] LUX collaboration, Improved limits on scattering of weakly interacting massive particles from reanalysis of 2013 LUX data, Phys. Rev. Lett. 116 (2016) 161301 [arXiv:1512.03506] [INSPIRE].

[82] SuPERCDMS collaboration, Projected sensitivity of the SuperCDMS SNOLAB experiment, Phys. Rev. D 95 (2017) 082002 [arXiv: 1610.00006] [InSPIRE].

[83] DARKSIDE collaboration, Low-mass dark matter search with the DarkSide-50 experiment, Phys. Rev. Lett. 121 (2018) 081307 [arXiv:1802.06994] [INSPIRE].

[84] E. Bernreuther, F. Kahlhoefer, M. Krämer and P. Tunney, Strongly interacting dark sectors in the early Universe and at the LHC through a simplified portal, JHEP 01 (2020) 162 [arXiv: 1907.04346] [INSPIRE].

[85] Y. Wu, K. Freese, C. Kelso, P. Stengel and M. Valluri, Uncertainties in direct dark matter detection in light of Gaia's escape velocity measurements, JCAP 10 (2019) 034 [arXiv: 1904.04781] [INSPIRE].

[86] The GAMBIT Dark Matter Workgroup collaboration, DarkBit: a GAMBIT module for computing dark matter observables and likelihoods, Eur. Phys. J. C 77 (2017) 831 [arXiv:1705.07920] [INSPIRE].

[87] R. Diamanti et al., Constraining dark matter late-time energy injection: decays and P-wave annihilations, JCAP 02 (2014) 017 [arXiv:1308.2578] [INSPIRE].

[88] A.J. Deason et al., The local high-velocity tail and the Galactic escape speed, Mon. Not. Roy. Astron. Soc. 485 (2019) 3514.

[89] XENON collaboration, First dark matter search results from the XENON1T experiment, Phys. Rev. Lett. 119 (2017) 181301 [arXiv:1705.06655] [INSPIRE].

[90] I. Goldman and S. Nussinov, Weakly interacting massive particles and neutron stars, Phys. Rev. D 40 (1989) 3221. 
[91] A. de Lavallaz and M. Fairbairn, Neutron stars as dark matter probes, Phys. Rev. D 81 (2010) 123521 [arXiv: 1004.0629] [INSPIRE].

[92] S.D. McDermott, H.-B. Yu and K.M. Zurek, Constraints on scalar asymmetric dark matter from black hole formation in neutron stars, Phys. Rev. D 85 (2012) 023519 [arXiv:1103.5472] [INSPIRE].

[93] C. Kouvaris and P. Tinyakov, Can neutron stars constrain dark matter?, Phys. Rev. D 82 (2010) 063531 [arXiv:1004.0586] [INSPIRE].

[94] C. Kouvaris, WIMP annihilation and cooling of neutron stars, Phys. Rev. D 77 (2008) 023006 [arXiv: 0708.2362] [INSPIRE].

[95] H. An, M.B. Wise and Y. Zhang, Strong CMB constraint on P-wave annihilating dark matter, Phys. Lett. B 773 (2017) 121 [arXiv:1606.02305] [INSPIRE].

[96] J.M. Cline and T. Toma, Pseudo-Goldstone dark matter confronts cosmic ray and collider anomalies, Phys. Rev. D 100 (2019) 035023 [arXiv:1906.02175] [INSPIRE].

[97] G. Bertone and M. Fairbairn, Compact stars as dark matter probes, Phys. Rev. D 77 (2008) 043515 [arXiv:0709.1485] [inSPIRE].

[98] M.I. Gresham and K.M. Zurek, Asymmetric dark stars and neutron star stability, Phys. Rev. D 99 (2019) 083008 [arXiv: 1809.08254] [inSPIRE].

[99] M. Baryakhtar et al., Dark kinetic heating of neutron stars and an infrared window on WIMPs, SIMPs and pure Higgsinos, Phys. Rev. Lett. 119 (2017) 131801 [arXiv: 1704.01577] [INSPIRE].

[100] N.F. Bell, G. Busoni and S. Robles, Capture of leptophilic dark matter in neutron stars, JCAP 06 (2019) 054 [arXiv: 1904.09803] [INSPIRE].

[101] J. Bramante, Dark matter ignition of type-IA supernovae, Phys. Rev. Lett. 115 (2015) 141301 [arXiv: 1505.07464] [INSPIRE].

[102] J.F. Acevedo and J. Bramante, Supernovae sparked by dark matter in white dwarfs, Phys. Rev. D 100 (2019) 043020 [arXiv: 1904.11993] [InSPIRE].

[103] H.B. Richer et al., Hubble space telescope observations of white dwarfs in the globular cluster m4, Astrophys. J. 451 (1995) L17 [astro-ph/9507109] [INSPIRE].

[104] L.R. Bedin et al., The end of the white dwarf cooling sequence in M4: an efficient approach, Astrophys. J. 697 (2009) 965 [arXiv:0903.2839] [INSPIRE].

[105] M. McCullough and M. Fairbairn, Capture of inelastic dark matter in white dwarves, Phys. Rev. D 81 (2010) 083520 [arXiv: 1001.2737] [INSPIRE].

[106] B. Dasgupta, A. Gupta and A. Ray, Dark matter capture in celestial objects: Improved treatment of multiple scattering and updated constraints from white dwarfs, JCAP 08 (2019) 018 [arXiv: 1906.04204] [INSPIRE].

[107] P.J.E. Peebles, Dark matter and the origin of galaxies and globular star clusters, Astrophys. $J 77$ (1984) 470.

[108] B.F. Griffen et al., Globular cluster formation within the Aquarius simulation, Mon. Not. Roy. Astron. Soc. 405 (2010) 375 [arXiv:0910.0310].

[109] M.A. Beasley, Globular cluster systems and galaxy formation, arXiv:2003.04093. 
[110] A.V. Kravtsov and O.Y. Gnedin, Formation of globular clusters in hierarchical cosmology, Astrophys. J. 623 (2005) 650 [astro-ph/0305199] [INSPIRE].

[111] D. Hooper, D. Spolyar, A. Vallinotto and N.Y. Gnedin, Inelastic dark matter as an efficient fuel for compact stars, Phys. Rev. D 81 (2010) 103531 [arXiv:1002.0005] [INSPIRE].

[112] I. Claydon et al., Spherical models of star clusters with potential escapers, Mon. Not. Roy. Astron. Soc. 487 (2019) 147 [arXiv:1903.05954].

[113] R. Krall and M. Reece, Last electroweak WIMP standing: pseudo-Dirac Higgsino status and compact stars as future probes, Chin. Phys. C 42 (2018) 043105 [arXiv:1705.04843] [INSPIRE].

[114] S. Tulin and H.-B. Yu, Dark matter self-interactions and small scale structure, Phys. Rept. 730 (2018) 1 [arXiv: 1705. 02358] [INSPIRE].

[115] S. Tulin, H.-B. Yu and K.M. Zurek, Resonant dark forces and small scale structure, Phys. Rev. Lett. 110 (2013) 111301 [arXiv:1210.0900] [INSPIRE].

[116] LUX collaboration, Results of a search for Sub-GeV dark matter using 2013 LUX data, Phys. Rev. Lett. 122 (2019) 131301 [arXiv:1811.11241] [INSPIRE].

[117] S. Cassel, Sommerfeld factor for arbitrary partial wave processes, J. Phys. G 37 (2010) 105009 [arXiv: 0903.5307] [INSPIRE].

[118] CDEX collaboration, Limits on light weakly interacting massive particles from the first $102.8 \mathrm{~kg} \times$ day data of the CDEX-10 experiment, Phys. Rev. Lett. 120 (2018) 241301 [arXiv: 1802.09016] [INSPIRE].

[119] I. Brivio and M. Trott, Radiatively generating the Higgs potential and electroweak scale via the seesaw mechanism, Phys. Rev. Lett. 119 (2017) 141801 [arXiv:1703.10924] [INSPIRE].

[120] I. Brivio and M. Trott, Examining the neutrino option, JHEP 02 (2019) 107 [arXiv: 1809. 03450] [INSPIRE].

[121] J.R. Ellis and M.K. Gaillard, Strong and weak CP-violation, Nucl. Phys. B 150 (1979) 141 [INSPIRE]. 